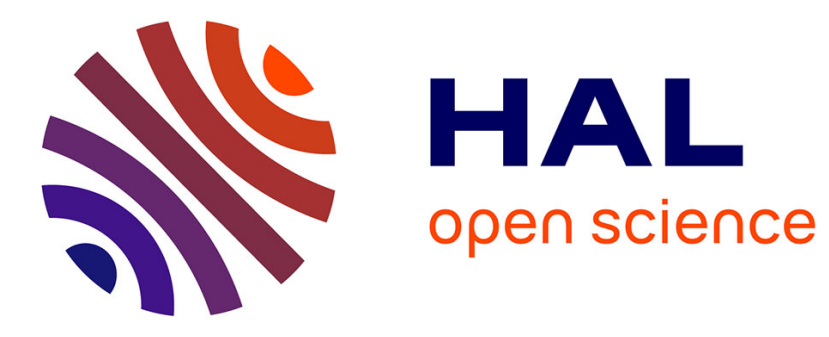

\title{
Direct Numerical Simulation of combustion near a carbonaceous surface in a quiescent flow
}

Adam M. Chabane, Karine Truffin, André Nicolle, Franck Nicoud, Olivier Cabrit, Christian Angelberger

\section{To cite this version:}

Adam M. Chabane, Karine Truffin, André Nicolle, Franck Nicoud, Olivier Cabrit, et al.. Direct Numerical Simulation of combustion near a carbonaceous surface in a quiescent flow. International Journal of Heat and Mass Transfer, 2015, 84, pp.130-148. 10.1016/j.ijheatmasstransfer.2014.12.017 . hal-01149022

\section{HAL Id: hal-01149022 \\ https://hal.science/hal-01149022}

Submitted on 6 May 2015

HAL is a multi-disciplinary open access archive for the deposit and dissemination of scientific research documents, whether they are published or not. The documents may come from teaching and research institutions in France or abroad, or from public or private research centers.
L'archive ouverte pluridisciplinaire HAL, est destinée au dépôt et à la diffusion de documents scientifiques de niveau recherche, publiés ou non, émanant des établissements d'enseignement et de recherche français ou étrangers, des laboratoires publics ou privés. 


\title{
Direct Numerical Simulation of combustion near a carbonaceous surface in a quiescent flow
}

\author{
Adam M Chabane ${ }^{\mathrm{a}, *}$, Karine Truffin ${ }^{\mathrm{a}}$, André Nicolle ${ }^{\mathrm{a}}$, Franck Nicoud ${ }^{\mathrm{c}}$, Olivier Cabrit ${ }^{\mathrm{b}}$, Christian Angelberger $^{\mathrm{a}}$ \\ ${ }^{a}$ IFP Energies nouvelles, 1-4 avenue de Bois-Préau, 92852 Rueil-Malmaison Cedex, France \\ ${ }^{b}$ Department of Mechanical Engineering, University of Melbourne, Victoria 3010, Australia \\ ${ }^{c}$ CNRS I3M 5149, University Montpellier 2, 34095 Montpellier, France
}

\begin{abstract}
In this work, unsteady gasification and oxidation of carbonaceous materials with gas-phase combustion under oxy-combustion conditions is studied. An original analytical solution is derived to validate the numerical species boundary condition for the heterogeneous surface reactions with frozen gas-phase. Direct Numerical Simulations accounting for multi-species diffusion and detailed chemistry with both species and energy boundary conditions taking into account the Stefan flux were subsequently performed to study combustion near a carbonaceous wall. Transition from gasification to solid carbon oxidation and gas-phase ignition is evidenced. The competition between homogeneous and heterogeneous reactions is discussed based on the analysis of the time-dependent surface and gas-phase species consumption and production rates. Transient histories of surface species as well as spatial profiles of gas-phase species, heat release and temperature are presented for two identified structures of gas-phase $\mathrm{CO} / \mathrm{O}_{2}$ reaction zone. In order to quantify the error induced by the Hirschfelder \& Curtiss approximation for the multi-species diffusion and to evaluate the importance of the Dufour and Soret terms, a library dedicated to the computation of complex diffusion phenomena has been used to rebuild a priori, species and Soret fluxes for mass diffusion as well as Fourier, species and Dufour diffusion heat fluxes. Eventually, characteristic times of gas and surface reactions and those corresponding to the switch between the two structures of the gas-phase reaction zone are obtained over a wide range of initial $\mathrm{O}_{2} / \mathrm{CO}_{2}$ concentrations.
\end{abstract}

Keywords:

Gasification, Oxidation, Ignition, DNS, Reactive surface

\footnotetext{
${ }^{*}$ Corresponding author

Email addresses: adam.chabane@ ifpen.fr (Adam

M Chabane), karine.truffineifpen.fr (Karine Truffin),

andre.nicolle@ifpen.fr(André Nicolle),

Preprint submitted to International journal of heat and mass transfer
}

franck.nicoudeuniv-montp2 frr (Franck Nicoud),

○.cabrit@unimelb.edu.au (Olivier Cabrit),

christian.angelberger@ifpen.fr

December 18, 2014 


\section{Introduction}

Oxy-fuel combustion is a cost-effective technology for $\mathrm{CO}_{2}$ capture from fuel fired furnaces. To control operating temperatures, flue gas is typically recycled into the combustion chamber, forming $\mathrm{O}_{2} / \mathrm{CO}_{2}$ atmosphere. Solid carbon ignition in such an atmosphere represents an issue not only for combustion technology but also for safety [1].

Despite the lower oxygen diffusivity in $\mathrm{CO}_{2}$ and the endothermicity of $\mathrm{CO}_{2}$ gasification (carbon conversion into carbon monoxide), the latter can actually lead to the enhancement of the overall carbon burning rate in oxy-fuel combustion $[2,3]$. Under steady-state conditions the effect of $\mathrm{CO}_{2}$ gasification on carbon conversion rate has been found to depend on $\mathrm{O}_{2}$ concentration [4]. The interaction between $\mathrm{CO}_{2}$ gasification and oxidation have been extensively studied $[5,6]$ especially at high pressure and high temperature [7] but remains poorly understood.

In $\mathrm{O}_{2}$-enriched conditions, gasification can contribute significantly to carbon consumption [8]. Although the sequential occurence [9] or respective importance [10] of homogeneous and heterogeneous ignition mechanisms have been evidenced experimentally, no detailed modeling work focused specifically, to our knowledge, on the interplay between $\mathrm{CO}$ production and consumption pathways under vitiated oxyfuel combustion. The contribution of heterogeneous and homogeneous reactions to carbon ignition is known to depend on the gas-phase composition [7] as well as on carbon particle size [11] and surface temperature [12, 13]. Makino et al. [14] studied the temporal evolution of oxidation and gasification contributions using global kinetics and transport and formulated a criterion for the existence of a $\mathrm{CO}$ flame. Zou et al [15] adressed the homogeneous and heterogeneous ignition processes of pulverized coal in oxy-fuel combustion using an Eulerian-Lagrangian approach with devolatilization kinetic sub-models. The impact of volatiles combustion processes, ambient gas temperatures, coal particle size and $O_{2}$ concentrations on the ignition type were assessed. It was concluded that heterogeneous ignition is more likely to occur for small coal particles and high $\mathrm{O}_{2}$ concentrations whereas homogeneous ignition launched by initial volatiles ignition is more likely to occur for large particles.

Many research groups still use simplified film models based on averaged diffusivity and global kinetics and neglect $\mathrm{CO}$ gas-phase conversion in the boundary layer. However, the predictivity of simplified film models need to be further improved [16, 17]. Lewtak et al. [18] showed that the use of an equimolar counterdiffusion model leads to the overprediction of mass transfer towards the particle. A recent review [7] stressed the need to account for multi-component diffusion effect in carbon oxy-fuel combustion. The sensitivity of carbon consumption to Stefan flow was shown to depend on the relative contributions of gasification and oxidation reactions. Stefan velocity can reach significant values when the surface coverages 
undergo drastic change during transient processes [19] which may give rise to gas-phase ignition [20]. Thus it is important to take into account this additional velocity induced by reactive walls.

Theoretical studies have been carried out using global chemistry, highlighting the differences between transient and steady-state behaviour [21], the impact of the relative importance of surface reactions on the shape of burning rate curve and the respective importance of homogeneous/heterogeneous chemistry and transport phenomena on particle ignition [22-24].

The aforementioned works reveal that transient homogeneous/heterogeneous carbon combustion data accounting for multi-species diffusion, detailed surface and gas-phase schemes with proper species and energy boundary conditions taking into account the Stefan flux remain scarce. Few studies emphasize the transient history of the heterogeneous/homogeneous carbon combustion. Hence, the objectives of this paper are twofold : An original analytical solution is first derived to develop and validate the numerical species boundary condition for the heterogenous surface reactions. Second, appropriate boundary conditions consistent with the conservation of gaseous/surface species and energy are applied to the direct numerical simulation of a configuration with both surface and gas-phase chemistry being active.

This study is organized as follows. First of all, an analytical solution is derived for a flow bounded by two reactive surfaces. Analytical solutions are then compared to numerical predictions for validation purposes (Section 3.1). Unsteady gasification and oxidation of carbonaceous materials coupled with gas-phase combustion under oxy-combustion conditions is analyzed through the DNS of a $\mathrm{CO} / \mathrm{O}_{2}$ combustion near a reactive carbonaceous surface in a quiescent flow (Section 3.2). Detailed and validated kinetics for surface reactions were used (Section 3.2.2). Transition from endothermic gasification to exothermic oxidation of the carbonaceous surface and $\mathrm{CO}$ gas-phase ignition is evidenced. The competition between homogeneous and heterogeneous reactions is discussed based on the analysis of the time-dependant surface and gas-phase species consumption and production rates. Transient histories of surface species concentrations as well as spatial profiles of gas-phase species, heat release and temperature are presented for two identified modes of gas-phase $C O$ reaction zone. The impact of the Hirschfelder \& Curtiss approximation on the results is assessed in Section 3.2.5 by post-processing the DNS data thanks to a complex transport library. At last, the impact of initial concentrations of $\mathrm{O}_{2}$ and $\mathrm{CO}_{2}$ on both competition between gas and surface reactions and characteristic times corresponding to the switch between the two structures of the gas-phase reaction zone is invistigated in Section 3.2.6. 


\section{Nomenclature \\ Dimensionless numbers \\ $D a$ \\ Damköhler number \\ Fo \\ Fourier number \\ Le \\ Lewis number \\ $\mathrm{Pr}$ \\ Prandtl number \\ $S c_{k}$ \\ Schmidt number of the $k^{\text {th }}$ species}

\section{Greek symbols}

\begin{tabular}{|c|c|}
\hline$\alpha_{j}$ & Pre-exponential factor of the $j^{\text {th }}$ reaction $(\mathrm{mol}, \mathrm{cm}, \mathrm{s})$ \\
\hline$\overline{\bar{\delta}}$ & Kronecker symbol \\
\hline$\varepsilon_{k, j}$ & $\begin{array}{l}\text { Surface coverage parameter for activation energy of the } k^{\text {th }} \text { species } \\
\text { involved in the } j^{\text {th }} \text { surface reaction }\end{array}$ \\
\hline$\eta_{k, j}$ & Surface coverage parameter for the pre-exponential factor of the $k^{\text {th }}$ species \\
\hline$\gamma_{j}$ & Sticking coefficient for adsorption reactions \\
\hline$\Gamma$ & Surface site density $\left(\right.$ mol. $\left.\mathrm{m}^{-2}\right)$ \\
\hline$\lambda$ & Heat conduction coefficient $\left(W \cdot m^{-1} \cdot K^{-1}\right)$ \\
\hline$\mu$ & Dynamic viscosity $(P a . s)$ \\
\hline$v_{k j}^{\prime \prime}, \quad v_{k j}^{\prime}$ & $\begin{array}{l}\text { Molar stoichiometric coefficient of the } k^{\text {th }} \text { species present as a product/reactant } \\
\text { in the } j^{\text {th }} \text { reaction }\end{array}$ \\
\hline$\Omega_{G}$ & Index set of gas-phase species involved in gas-phase and surface reactions \\
\hline$\Omega_{s}$ & Index set of surface species involved in surface-phase reactions \\
\hline$\dot{\omega}_{k}$ & Rate of production by gas-phase reactions of the $k^{\text {th }}$ species $\left(k g \cdot \mathrm{m}^{-3} \cdot \mathrm{s}^{-1}\right)$ \\
\hline$\dot{\omega}_{T}$ & Gas-phase heat release in $\left(J \cdot m^{-3} \cdot s^{-1}\right)$ \\
\hline$\rho$ & Gas-phase density $\left(k g \cdot m^{-3}\right)$ \\
\hline$\sigma_{k}$ & Number of sites that each surface species occupies \\
\hline$\overline{\bar{\tau}}$ & Viscous stress tensor $\left(k g \cdot m^{-1} \cdot s^{-2}\right)$ \\
\hline$\theta_{k}$ & Surface species site fraction \\
\hline$\tau$ & Dimensionless time \\
\hline$\chi_{k}$ & Thermal diffusion ratios \\
\hline
\end{tabular}




\section{Roman letters}

\begin{tabular}{|c|c|}
\hline$C_{k}$ & Molar concentration of the $k^{\text {th }}$ surface species $\left(\right.$ mol. $\left.\mathrm{m}^{-2}\right)$ \\
\hline$C_{p, k}, C_{v, k}$ & Heat capacity at constant pressure and constant volume $\left(J . \mathrm{kg}^{-1} \cdot \mathrm{K}^{-1}\right)$ \\
\hline $\mathcal{D}$ & Index set of space dimensions \\
\hline$D_{k}$ & Mass diffusion coefficient of the $k^{t h}$ gas-phase species $\left(m^{2} \cdot s^{-1}\right)$ \\
\hline$E$ & Total non-chemical energy $\left(J . \mathrm{kg}^{-1}\right)$ \\
\hline$E_{j}$ & Activation energy of the $j^{\text {th }}$ reaction $\left(\mathrm{J} . \mathrm{mol}^{-1}\right)$ \\
\hline$h_{s, k}, h_{k}$ & Sensible and total enthalpy of the $k^{\text {th }}$ species $\left(J \cdot \mathrm{kg}^{-1}\right)$ \\
\hline$\Delta h_{f, k}^{o}$ & Standard enthalpy of formation of the $k^{\text {th }}$ gas-phase species $\left(J \cdot \mathrm{kg}^{-1}\right)$ \\
\hline $\overrightarrow{J_{k}}$ & Diffusive flux of the $k^{\text {th }}$ gas-phase species $\left(k g \cdot \mathrm{m}^{-2} \cdot \mathrm{s}^{-1}\right)$ \\
\hline$k_{f, j}, k_{f, j}^{s}$ & Forward rate constants of the $j^{\text {th }}$ gas-phase and surface reaction $(\mathrm{mol}, \mathrm{cm}, \mathrm{s})$ \\
\hline$K_{e q}$ & Equilibrium constant \\
\hline$l_{\text {diff }}$ & Characteristic diffusion length within a chemical time $\tau_{\text {chem }}$ \\
\hline$m_{c}$ & Surface carbon mass $(k g)$ \\
\hline$\dot{m}$ & Convected mass rate at the wall $\left(k g . m^{-2} \cdot s^{-1}\right)$ \\
\hline$n$ & Reaction order \\
\hline$p$ & Thermodynamic pressure $(\mathrm{Pa})$ \\
\hline$\vec{q}$ & Heat flux $\left(J . m^{-2} . s^{-1}\right)$ \\
\hline$Q_{j}$ & Reaction rate of the $j^{\text {th }}$ gas-phase reaction $\left(\right.$ mol. $\left.\mathrm{m}^{-3}\right)$ \\
\hline$Q_{s, j}$ & Reaction rate of the $j^{\text {th }}$ surface reaction $\left(\right.$ mol. $\left.m^{-2}\right)$ \\
\hline$\dot{s}_{k}$ & Rate of production by surface reactions of the $k^{\text {th }}$ species $\left(k g \cdot \mathrm{m}^{-2} \cdot \mathrm{s}^{-1}\right)$ \\
\hline$R$ & Universal gas constant $\left(J . m o l^{-1} K^{-1}\right)$ \\
\hline$T$ & Temperature $(K)$ \\
\hline$\vec{u}$ & Convection velocity field $\left(m \cdot s^{-1}\right)$ \\
\hline$U_{\text {Stefan }}$ & Stefan velocity $\left(m \cdot s^{-1}\right)$ \\
\hline$\vec{V}_{k}$ & Diffusion velocity field of the $k^{\text {th }}$ gas-phase species $\left(m \cdot s^{-1}\right)$ \\
\hline$W_{k}, W$ & Molar weight of the $k^{\text {th }}$ species and gas-phase mixture $\left(\mathrm{kg} . \mathrm{mol}^{-1}\right)$ \\
\hline$w$ & Index denoting for values of scalars or vector components at the wall \\
\hline$X_{k}, Y_{k}$ & Mole and mass fraction of the $k^{\text {th }}$ gas-phase species \\
\hline
\end{tabular}




\section{Modelling approach}

\subsection{Surface chemistry formalism}

\subsubsection{Surface reactions kinetics}

Only reactions occuring on the top layer of the solid are considered and modeled through an open site formalism; bulk reactions are not considered. For each species, the surface reaction rate of production $\dot{s}_{k}$ is the sum of rates of surface reactions :

$$
\dot{s}_{k}=W_{k} \sum_{j \in \mathcal{M}_{s}}\left(v_{k j}^{\prime \prime}-v_{k j}^{\prime}\right) Q_{s, j} \forall k \in\left\{\Omega_{g} \cup \Omega_{s}\right\} .
$$

where $Q_{s, j}$ represents the rate of progress of the $j^{\text {th }}$ surface reaction and is written according to the mean field approximation [25]:

$$
Q_{s, j}=k_{f, j}^{s}\left(\prod_{k \in \Omega_{G}}\left(\rho_{w}^{g} Y_{k, w} / W_{k}\right)^{v_{k j}^{\prime}}\right)\left(\prod_{k \in \Omega_{s}}\left(\Gamma \theta_{k}\right)^{v_{k j}^{\prime}}\right)
$$

In Eq. (2), $\theta_{k}$ are surface-species site fractions and $k_{f, j}^{s}$ is the forward reaction constant of the $j^{\text {th }}$ surface reaction. Only mono-directional steps are considered in this study. The reaction constants are evaluated according to the surface reaction type. The $j^{\text {th }}$ surface reaction constant is given by :

$$
k_{f, j}^{s}=A_{s, j}^{c o v} T_{w}^{\beta_{s, j}} \exp \left(\frac{-E_{s, j}^{c o v}}{R T_{w}}\right)
$$

where $A_{s, j}^{c o v}$ and $E_{s, j}^{c o v}$ are respectively the coverage dependent pre-exponential factor and activation energy that can be written as follows:

$$
\ln \left(A_{s, j}^{c o v}\right)=\ln \left(A_{s, j}\right)+\sum_{k \in \Omega_{s}} \eta_{k, j} \theta_{k}
$$

and

$$
E_{s, j}^{c o v}=E_{s, j}+\sum_{k \in \Omega_{s}} \varepsilon_{k, j} \theta_{k}
$$

Assuming the adsorption to be activated, the parameters $\beta_{s, j}, E_{s, j}^{c o v}$ and $A_{s, j}^{c o v}$ become [26-28] :

$\beta_{s, j}=\frac{1}{2}, E_{s, j}^{c o v}=0, A_{s, j}^{c o v}=\gamma_{j}\left(\frac{\prod_{k \in \Omega_{s}} \sigma_{k}^{v_{k, j}^{\prime}}}{\Gamma^{m}}\right) \sqrt{\frac{R}{2 \pi W_{g, a d s}}}$

where $\sigma_{k}$ is the number of sites that each adsorbed species occupies, $m$ corresponds to the number of surface species involved in the surface reaction and $W_{g, a d s}$ is the gas-phase species molar weight. $\theta_{k}$ is related to the surface mole concentration of the $k^{\text {th }}$ surface species, $C_{k}$, as follows :

$$
\theta_{k}=\frac{C_{k} \sigma_{k}}{\Gamma}
$$

The equation describing the variation of surface coverage [19] is based on the assumption that site density $\Gamma$ remains constant, diffusion inside the solid is neglected as surface local diffusion is considered to be very fast with respect to reactions and every $k^{\text {th }}$ surface species occupies only one site, i.e, $\sigma_{k}=1$ :

$$
\frac{\partial \theta_{k}}{\partial t}=\frac{\dot{s}_{k}}{\Gamma W_{k}} \forall k \in \Omega_{s}
$$

The surface site fractions correspond to a single mono-layer which implies that their sum equals unity.

$$
\sum_{k \in \Omega_{s}} \theta_{k}+\theta_{\text {void }}=1
$$

where $\theta_{\text {void }}$ corresponds to the fraction of void sites. 


\subsubsection{Accounting for surface chemistry through boundary conditions}

The flow solver used for this study is a conservative finite-volume based and known as AVBP [29]. It has been extensively validated over a wide range of applications [30-33]. In Appendix A, the set of the unfiltered equations used in Direct Numerical Simulations (DNS) governing the evolution of a compressible flow with gas-phase chemical reactions is described. The modeling of transport coefficients, diffusion and gas-phase kinetics are also available.

AVBP is a gas-phase solver whereas surface reactions correspond to chemical phenomena occuring at the wall. Therefore, the interaction of the latter with the gas-phase scalars was accounted for through boundary conditions. Heterogeneous reactions occuring at the reactive wall consume or produce gas-phase species. This consumption or production leads to an additional velocity at the interface while it releases or adsorbs heat because of solid-fluid reactions that are not necessarily athermal. Hence, surface chemistry impacts gas-phase species mass fractions, energy and momentum. Details of the approach giving the mass and energy balance at a reactive interface with the associated boundary conditions are provided in Appendix B. $w$ is the index refering to variables at the wall and $\vec{n}$ is the vector normal to the wall and pointing toward the gas-phase. Gas-phase species are adsorbed and desorbed through reactions at the reactive wall thus resulting in a mass flux which is convected at a local speed orthogonal to the wall called the Stefan velocity:

$$
\vec{u}_{w}=U_{\text {Stefan }} \vec{n}
$$

$\vec{u}_{w}$ is the velocity vector at the reactive wall. The Stefan velocity is obtained from the species mass conservation balance at the reactive interface.

$$
\left(\rho_{w} V_{k, n, w} Y_{k, w}\right)+\left(\rho_{w} U_{\text {Stefan }} Y_{k, w}\right)=\dot{s}_{k}
$$

$V_{k, n, w}$ refers to the normal diffusion velocity at the reactive wall. Summing Eq. (11) over all the gas-phase species and considering the mass conservation, an expression for the wall-normal Stefan velocity is obtained:

$$
U_{\text {Stefan }}=\frac{1}{\rho_{w}}\left(\sum_{k \in \Omega_{G}} \dot{s}_{k}\right)=\frac{\dot{m}}{\rho_{w}}
$$

where $\dot{m}$ is the convected mass-rate at the wall.

Surface chemistry impacts the gas-phase species mass fraction at the reactive wall. The prescribed normal gradient noted $\vec{\nabla} Y_{k, w}^{B C} \cdot \vec{n}$ is obtained by expressing in the mass balance given in Eq. (11) the diffusion velocity in terms of species mass fractions gradients by using the Hirschfelder \& Curtiss approximation [34, 35] whose impact is evaluated in Section 3.2.5. Hence, it becomes possible to relate the species mass fraction at the wall $Y_{k, w}$ to its normal gradient at the boundary surface :

$$
\begin{aligned}
\vec{\nabla} Y_{k, w}^{B C} \cdot \vec{n} & =\frac{Y_{k, w}}{D_{k} \rho_{w}} \sum_{l \in \Omega_{G}} \dot{s}_{l}+\frac{Y_{k, w}}{D_{k}} \vec{V}_{w}^{c o r r e c} \cdot \vec{n} \\
& +Y_{k, w} W_{w}\left(\sum_{l \in \Omega_{\text {gas }}} \frac{1}{W_{l}} \vec{\nabla} Y_{l, w} \cdot \vec{n}\right)-\frac{\dot{s}_{k}}{\rho_{w} D_{k}}
\end{aligned}
$$


Eq. (13) corresponds to the boundary condition for species mass fractions and constitutes a non-linear system of equations which is solved thanks to an iterative method [36, 37]. In the following, the energy boundary condition accounting for surface chemistry is developed. Surface chemistry in non-athermal cases impacts the wall temperature since surface reactions may be endothermic or exothermic while the reactive interface undergoes heat exchange including gas-phase thermal diffusion and convection, species diffusion, viscous heat and surface reactions heat release involving both surface and gas-phase species. The heat balance at the reactive wall reads:

$$
\begin{aligned}
\lambda^{g} \vec{\nabla} T_{w}^{g} \cdot \vec{n} & =\sum_{k \in\left\{\Omega_{G} \cup \Omega_{S}\right\}} h_{k, w} \dot{s}_{k}-(\overline{\bar{\tau}} \vec{u})_{w} \cdot \vec{n} \\
& +\frac{\dot{m}}{2} \sum_{j \in \mathcal{D}} u_{j, w}^{2}+\lambda^{s} \vec{\nabla} T_{w}^{s} \cdot \vec{n}
\end{aligned}
$$

Eq. (14) constitutes the heat flux balance that accounts for surface chemistry impact on energy. The balance enables to write the heat flux to be prescribed to the boundary condition $q_{n, w}^{B C}$ as shown in Eq. (17). The rate of production/consumption of species due to gas-phase reactions is set to zero at the wall so that no gas-phase reactions occur at the reactive surface :

$$
\dot{\omega}_{k, w}=0
$$

The resulting heat release due to gas-phase reaction presents zero values at the wall :

$$
\dot{\omega}_{T, w}=0
$$

The conditions of Eqs. $(15,16)$ are not related to equilibrium assumptions. $\dot{\omega}_{k}$ and $\dot{\omega}_{T}$ are set to zero so that the species and energy boundary conditions are consistent with Eq. (B.5). Therefore, the near-wall grid resolution chosen for this study ( $\sec 3.2 .1$ ) is judged fair enough to ensure these conditions.

$$
\begin{aligned}
& {\overrightarrow{q_{w}}}^{B C} \cdot \vec{n}=\underbrace{-\sum_{k \in \Omega_{S}} h_{k} \dot{s}_{k}}_{\text {Surface heat release due to surface species }} \\
& -\underbrace{\sum_{k \in \Omega_{G}} \Delta h_{f}^{o} \dot{s}_{k}}_{\text {Surface heat release due to gas-phase species }} \\
& -\underbrace{\rho_{w} \sum_{k \in \Omega_{G}} U_{S t e f a n} h_{s, k, w} Y_{k, w}}_{\text {Sensible enthalpy transport by Stefan flux }} \\
& -\underbrace{\frac{\dot{m}}{2} \sum_{j \in \mathcal{D}} u_{j, w}^{2}}_{\text {Kinetic energy }}+\underbrace{(\overline{\bar{\tau}} \vec{u})_{w} \cdot \vec{n}}_{\text {Viscous heat }}-\underbrace{\lambda^{s} \vec{\nabla} T_{w}^{s} \cdot \vec{n}}_{\text {Solid heat diffusion }}
\end{aligned}
$$

A Dirichlet condition is applied to the velocity vector at the wall in order to impose the Stefan velocity as shown in Eq. (18).

$$
\vec{u}_{w}^{c o r}=U_{\text {Stefan }} \vec{n}
$$

Where $\vec{u}_{w}^{\text {cor }}$ is the corrected velocity at the wall. The normal gas-phase species mass fraction gradients and the heat flux are imposed numerically at the reactive surface as shown in Eqs. $(19,20)$. First, the numerical scheme predicts the gradient of the $k^{\text {th }}$ species mass fraction $\vec{\nabla} Y_{k, w}^{p}$ and the heat flux $\vec{q}_{w}^{p}$ at the reactive wall. These gradients are then modified by imposing the 
prescribed normal gradients and the normal heat flux $\vec{\nabla} Y_{k, w}^{B C} \cdot \vec{n}$ and $\vec{q}_{w}^{B C} \cdot \vec{n}$ obtained respectively from the mass and energy balance at the reactive interface given in Eqs. $(11,14)$.

$$
\begin{gathered}
\vec{\nabla} Y_{k, w}^{c o r}=\vec{\nabla} Y_{k, w}^{p}+\left(\vec{\nabla} Y_{k, w}^{B C} \cdot \vec{n}-\vec{\nabla} Y_{k, w}^{p} \cdot \vec{n}\right) \cdot \vec{n} \\
\vec{q}_{w}^{c o r}=\vec{q}_{w}^{p}+\left(\vec{q}_{w}^{B C} \cdot \vec{n}-\vec{q}_{w}^{p} \cdot \vec{n}\right) \cdot \vec{n}
\end{gathered}
$$

Where $\vec{\nabla} Y_{k, w}^{c o r}$ and $\vec{q}_{w}^{c o r}$ are the corrected species mass fraction gradient and heat flux, $\vec{\nabla} Y_{k, w}^{p} \cdot \vec{n}$ and $\vec{q}_{w}^{p} \cdot \vec{n}$ correspond to the normal component of the $k^{\text {th }}$ species mass fraction and the heat flux predicted by the numerical scheme.

\section{Results}

\subsection{Analytical solution for wall-bounded flow with carbonaceous wall gasification}

In this section the purpose is to derive an original analytical solution to validate the numerical species boundary condition for the heterogeneous surface reactions with frozen gas-phase. This solution can be used to understand the physics and easily analyze the influence of some physical parameters onto the studied phenomena. Moreover, analytical solutions are of wide interest for experimentalists or computational fluid dynamics users to validate their experimental/numerical procedure. Only the global approach is described and the final solution is given. The complete development is provided as a supplementary material in Appendix
C. It is sometimes possible to find the analytical solution of the system of partial differential equations that governs the physics of simple two-dimensional laminar flows. An example is the Poiseuille flow configuration which allows to give the analytical velocity/temperature profiles under the assumption of streamwise-developed steady flow [38]. Introducing the transpiring surface effects, Koh and Hartnett [39] have shown that the analytical solution can also be recovered for flow over permeable wedges. Hamza [40] also presented the solution of channel flow with transpiring and moving walls for biomechanics-fluid interest. Concerning reacting wall effects, Rosner [41] introduced a set of solutions for surface catalyzed problems. Duan and Martín [42] also mase use of an analytical solution for a diffusion-tube sidearm reactor in order to validate their numerical simulation of airflow over purely catalytic wall. To our knowledge, surface ablation has received little attention in the literature. Libby \& Blake [8] developed an analytical solution for ablation but their case of study was purely diffusive. A special attention is given to the configuration set-up which allows to seek a steady-state solution. The idea consists in considering a channel problem of which the lower surface undergoes gaseous species production and solid carbon recession whereas the upper one encounters carbon deposition. Within this framework, the velocity and molar fraction profiles are derived and discussed. Global surface reactions and kinetic models are used, the purpose in this section being to seek numerical validation and not 
kinetic accuracy. The surface consumption has an effect on the flow since the products of the heterogeneous reactions include atoms that were originally embedded in the wall lattice. As a consequence, the surface geometry is changed according to the reaction rates at the surface. This feature makes the derivation of the solution more challenging, especially when a steady-state solution is sought for. As presented hereafter, the configuration set-up has to be properly thought to handle this undesirable effect. To simplify the derivation, one seeks for a solution that satisfies the following assumptions:

- two-dimensional laminar flow,

- steady state,

- channel flow developed in streamwise direction (periodic boundary conditions),

- non-reacting binary mixture (frozen state),

- constant Schmidt numbers,

- pure gasification (no pyrolysis or sublimation of the wall material),

- isothermal gasified surface with finite reaction rates,

- constant pressure and temperature.

In such a configuration, a special attention must be given to the chemical scheme retained for the heterogeneous surface reactions. Indeed, if the same scheme is applied to the two surfaces, this leads to a flow that similarly behaves at the wall surfaces. This means that the two convective wall normal velocity vectors induced by surface gasification are pointing towards opposite directions. This translates into an overall increase (or decrease) of mass that is not compatible with the steady state assumption. For this reason, one sets up a configuration in which one of the surface is consumed by a reaction while the reverse reaction takes place at the facing surface so mass increase or decrease in the domain is avoided. The set-up retained is summarized in Fig. (1). The flow is supposed to evolve in a streamwise periodic channel between two plates separated by the distance $h$. The wall normal direction is referenced by the letter $y$ whereas $x$ is used for the streamwise direction.

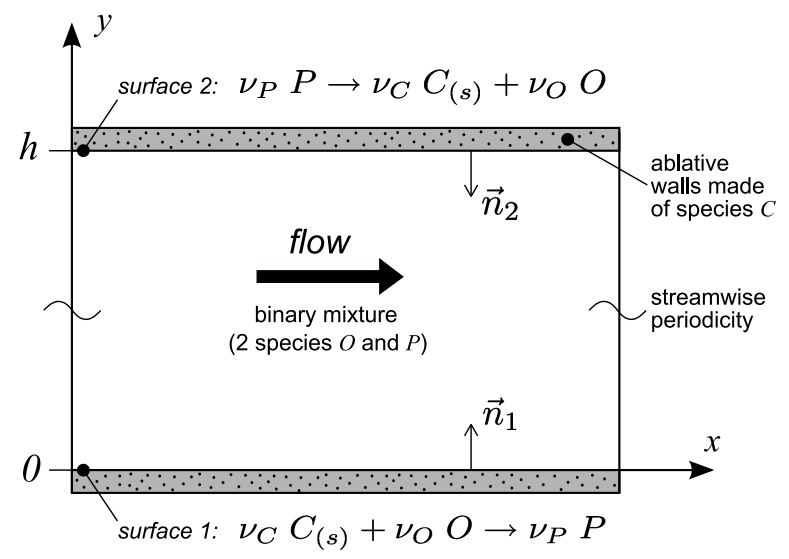

Figure 1: Sketch of the configuration.

Making use of the assumption of steady developed non-reacting flow, one can write the multicomponent Navier-Stokes mass/species/momentum conservation equations as:

$$
\frac{\partial(\rho v)}{\partial y}=0
$$




$$
\begin{gathered}
\frac{\partial}{\partial y}\left(\rho\left(v+V_{k, y}\right) Y_{k}\right)=0, \quad k \in\{1,2\} \\
\frac{\partial(\rho u v)}{\partial y}=\frac{\partial \tau_{x y}}{\partial y}+\mathcal{S}
\end{gathered}
$$

Where $u$ the streamwise velocity component, $v$ the wall normal velocity component, $Y_{k}$ the mass fraction of species $k, V_{k, y}$ the wall normal diffusion velocity of species $k, \tau_{x y}=\mu d u / d y$ the shear stress, and $\mathcal{S}$ is the source term driving the flow. Note that this term must be added because the periodicity condition along $x$ imposes $\partial p / \partial x=0$. Finally, the perfect gas equation of state is used to close the problem, which supposes that no real gas effects are considered. Moreover, the general formulation of species conservation balance at the reacting surfaces reads:

$$
\left(\rho_{j} \vec{u}_{j} Y_{k, j}+\rho_{j} \vec{V}_{k, j} Y_{k, j}\right) \cdot \vec{n}_{j}=\dot{s}_{k, j}
$$

Index $j$ refers to the reactive surface number, $j=1$ being the lower surface whereas $j=2$ corresponds to the upper one. $\vec{n}_{j}$ is the unitary wall normal vector at surface $j$ directed towards the gaseous domain as shown in Fig. (1). $\dot{s}_{k, j}$ is the mass production rate of species $k$ defined as $\dot{s}_{k, j}=\overline{v_{k, j}} W_{k} Q_{j}$ with $\overline{v_{k, j}}$ the algebraic stoichiometric coefficient of the $k^{\text {th }}$ species at the $j^{\text {th }}$ surface (positive for produced species, negative for consumed species). Under these conditions described in Fig. (1), one seeks for an analytical solution for the independent variables $u(y), v(y), Y_{k}(y)$ (or equivalently $X_{k}(y)$ via the relation $\left.X_{k}=Y_{k} W / W_{k}\right)$. The integration of Eq. (24) leads to two solutions given in Eq. (25) depending on the values taken by the stochiometric numbers $v_{O}$ and $v_{P}$ refering respectively to the oxidizer (O) and the product(P).

$X_{O}(y)=\left(X_{O, 1}-\frac{v_{O}}{v_{O}-v_{P}}\right) \exp \left[\eta \frac{S c_{O} \dot{m}}{\mu} y\right]+\frac{v_{O}}{v_{O}-v_{P}}$

for $v_{O} \neq v_{P}$

$X_{O}(y)=-\frac{S c_{O} \dot{m}}{\mu} \frac{W_{P}}{W_{O}-W_{P}} y+X_{O, 1}$

for $v_{O}=v_{P}$

The mole fraction of $P$ is then given by the relation $X_{P}(y)=1-X_{O}(y)$. Here $X_{O, 1}$ is the molar fraction of species $O$ at surface $1, \dot{m}$ correspond to the total mass convected mass-rate at the reactive surfaces, $S c_{O}$ is the Schmidt number of the oxidizer species and $\eta=\frac{W_{P}\left(v_{O}-v_{P}\right)}{v_{O} W_{O}-v_{P} W_{P}}$. Concerning the determination of the streamwise velocity profile, one introduces the newtonian fluid relation $\tau_{x y}=\mu d u / d y$ into Eq. (23) which gives $\rho v \quad d u / d y-\mu d^{2} u / d y^{2}=\mathcal{S}$. Since $\mu$ is constant under the approximation of constant temperature, and because $\rho(y) v(y)=\dot{m}$ is constant according to the continuity equation given in Eq. (21), the latter differential equation is merely of second order with constant coefficients. Its integration with the boundary conditions $u(0)=u(h)=0$ leads to the following solution:

$$
u(y)=\frac{\mathcal{S}}{\dot{m}}\left[\frac{h}{\mathrm{e}^{\frac{\dot{m}}{\mu} h}-1}\left(1-\mathrm{e}^{\frac{\dot{m}}{\mu} y}\right)+y\right]
$$

It is interesting to notice that if $\dot{m}$ tends to zero, the use of second-order Taylor series in Eq. (26) allows to demonstrate that the streamwise velocity profile 
tends to the classical Poiseuille one, namely $u(y)=$ $\mathcal{S} /(2 \mu)\left(y h-y^{2}\right)$. Finally, the maximum streamwise velocity is expected to be observed at the wall normal distance which differs from a classical Poiseuille profile for which the maximum velocity is observed at the channel half-height. This deviation is only due to the wall normal mass flow rate and non-sensitive to the driving pressure gradient. Note also that making use of Taylor series (for $\dot{m} \rightarrow 0$ ) it is easy to show that $\left.y\right|_{u_{\max }} \rightarrow h / 2$.

$$
\left.y\right|_{u_{\max }}=\frac{\mu}{\dot{m}} \ln \left[\frac{\mu}{h \dot{m}}\left(\mathrm{e}^{\frac{\dot{m}}{\mu} h}-1\right)\right]
$$

The analytical solution is now discussed in the case of carbon $\mathrm{CO}_{2}$ gasification. Three cases presented in Table 1 , for which $h=1 \mathrm{~mm}$ and $\mathcal{S}=1 \mathrm{kPa} / \mathrm{m}$. The transport coefficients are determined thanks to the EGlib library [43]. All cases represent a realistic solution for which the surface reaction has a finite-rate, in other words, the concentration of the oxidizing species is different from zero at surface 1 and different from unity at surface 2 . In order to ensure a balance between both forward and backward reactions occuring respectively at surface 1 and 2, constants of reactions are set equal, i.e, $K_{1}=K_{2}$.

The comparisons of analytical and numerical solutions for the wall normal velocity component, the mass fraction of the oxidizing species and the streamwise velocity are presented in Figs. (2-4) for $\mathrm{CO}_{2}$ gasification. It is noticed that the wall normal distance for which the maximum velocity is observed $\left.y\right|_{u_{\max }}$ is identical to the channel half-height $h / 2$. Moreover, the fluid encouters the same wall shear stress at both surfaces, i.e, $\tau_{w, 2} / \tau_{w, 1} \approx 1$. These cases correspond to values of mass rates that are too low to modify the streamwise velocity profiles significantly (Table 2). As a result, these profiles are very close to the impermeable wall solution. The analytical solutions are identical to the numerical ones, showing that the boundary conditions taking into account the Stefan velocity are able to predict correctly the influence of surface reactions on gas-phase species and momentum given respectively in Figs. $(3,4)$.

\subsection{Unsteady combustion near a carbonaceous surface}

An original analytical solution was derived in the previous section to develop and validate the numerical species boundary condition for the heterogeneous surface reactions. In this section, appropriate boundary conditions consistent with the conservation of gaseous/surface species and energy are applied to the direct numerical simulation of a configuration with both surface and gas-phase chemistry being active.

\subsubsection{Numerical setup}

The considered problem is purely $1 \mathrm{D}$, variations occuring exclusively between the upper and lower surface and all fields being homogeneous in the $y$ and $z$ directions because of periodicities. The configuration studied is a parallelepipedic channel containing initially a quiescent and homogeneous 
Table 1: Parameters of the test cases.

\begin{tabular}{cccccccccc} 
case & Oxidizer $(O)$ & Product $(P)$ & $v_{O}$ & $v_{P}$ & $S c_{O}$ & $S c_{P}$ & $K_{1}=K_{2}\left({\left.\mathrm{~kg} . \mathrm{m}^{-1} \cdot \mathrm{mol}^{-1} \cdot \mathrm{s}^{-1}\right)[8]}\right.$ & $P(\mathrm{~atm})$ & $T(\mathrm{~K})$ \\
\hline 1 & $\mathrm{CO}_{2}$ & $C O$ & 1 & 2 & 0.92 & 0.59 & $6.92 \times 10^{-3}$ & 1 & 1400 \\
2 & $\mathrm{CO}_{2}$ & $C O$ & 1 & 2 & 0.92 & 0.59 & $5.2 \times 10^{-2}$ & 1 & 1600 \\
3 & $\mathrm{CO}_{2}$ & $C O$ & 1 & 2 & 0.92 & 0.59 & 0.49 & 1 & 1900
\end{tabular}

Table 2: Characteristic values of the test cases.

\begin{tabular}{cccc} 
case & $\dot{m}$ & $\frac{\left.y\right|_{u_{\max }}}{h}$ & $\tau_{w, 2} / \tau_{w, 1}$ \\
\hline 1 & $3.87 \times 10^{-4}$ & 0.5 & 1 \\
2 & $2.16 \times 10^{-3}$ & 0.5 & 1.01 \\
3 & $8.88 \times 10^{-3}$ & 0.5 & 1.052
\end{tabular}

$\mathrm{O}_{2} / \mathrm{CO}_{2}$ mixture. Solid carbon consumption and $\mathrm{CO} / \mathrm{CO}_{2}$ production through surface gasification and oxidation takes place at the lower wall, whereas the upper surface corresponds to an outlet ensuring a constant pressure within the whole channel. The channel height $h$ is higher than the distance $l_{\text {diff }}=$ $\sqrt{\left.\tau_{\text {chem }}(v / \overline{S c})\right|_{t=0}}$ characterizing diffusion within a chemical time $\tau_{\text {chem }}$. The latter is evaluated in a homogeneous adiabatic reactor provided with identical initial thermodynamic and thermo-chemical conditions as the present studied case. The corresponding value of $\tau_{\text {chem }}$ coincides with complete depletion of surface carbon, i.e, $\theta_{C(S)}=0 .\left.(v / \overline{S c}) \quad\right|_{t=0}$ refers to the ratio of kinematic viscosity and the average Schmidt number of the mixture. According to this approach, the initial Damköhler number $(D a)$ is much larger than unity which makes the combustion process overall diffusion-limited.

$$
D a=\left(\frac{h}{l_{\text {diff }}}\right)^{2}>>1
$$

The surface over volume ratio $A_{\text {wall }} / V$ ensures that the gas and surface reactions contributions to $\mathrm{CO}_{2}$ and $\mathrm{O}_{2}$ consumption and production are of the same order of magnitude which implies that the surface species consumption proceeds at a rate as fast as gas-phase chemistry. $A_{\text {wall }}$ being the chemically active surface area of the lower wall ( Fig. (5) ), the ratio $A_{\text {wall }} / V$ reads :

$$
\frac{A_{\text {wall }}}{V}=\frac{a^{2}}{a^{2} h}=\frac{1}{h}
$$

Variables intialization is spatially homogeneous, Table 3 summarizes the initial concentrations and thermodynamic parameters for the reference case. The transport coefficients are determined using the EGLIB library [43]. An adiabatic condition is applied to the lower reactive surface which means that the 


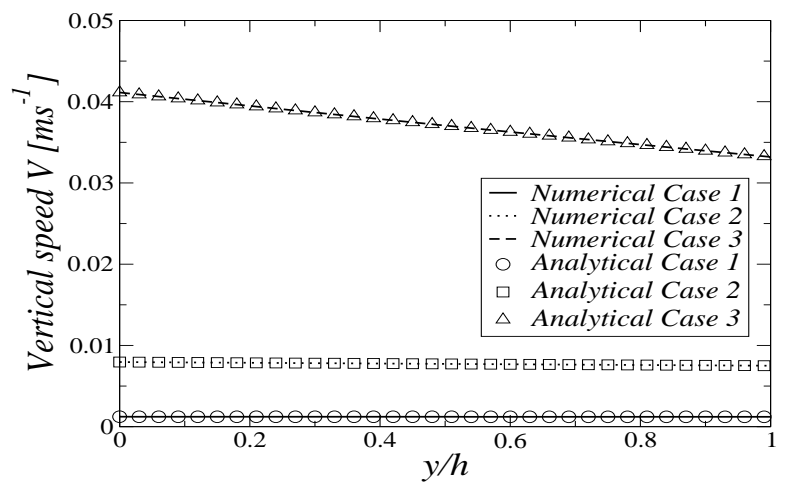

Figure 2: Analytical and numerical spatial profiles of the vertical velocity

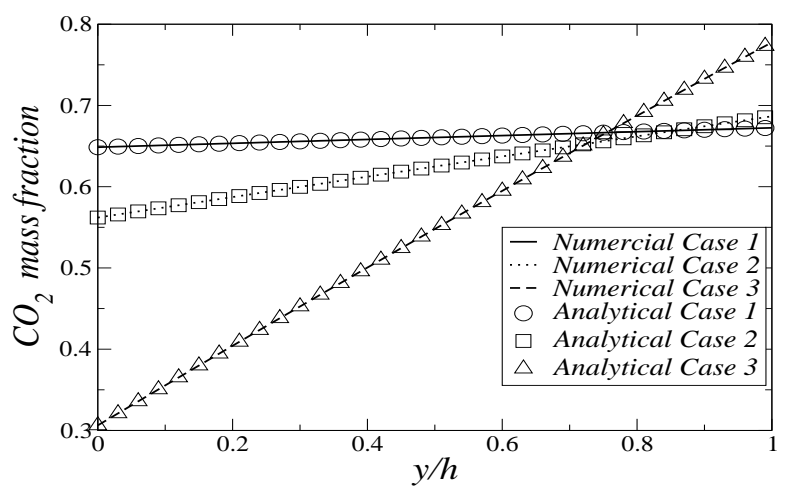

Figure 3: Analytical and numerical spatial profiles of species mass fractions

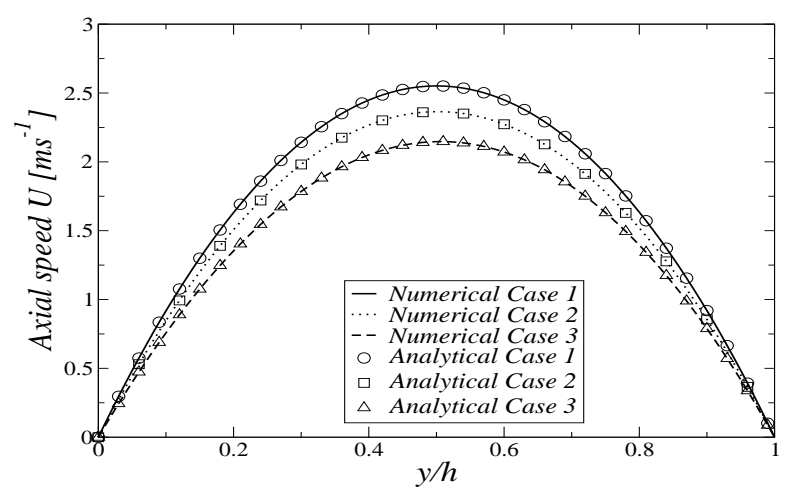

Figure 4: Analytical and numerical spatial profile of the streamwise velocity

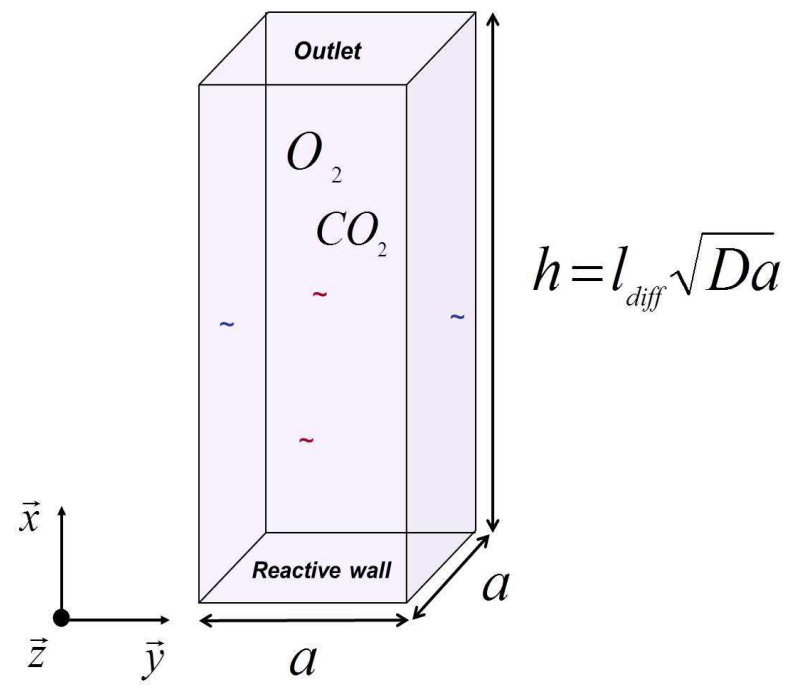

Figure 5: Schematic drawing of the setup

temperature of the reactive wall presents temporal variations based on the heat flux balance given in Eq. (14). The condition is called adiabatic in the sense that no thermal conduction is accounted for within the solid, which means that $\lambda^{s} \vec{\nabla} T_{w}^{s} \cdot \vec{n}=0$. The latter expression implies that the prescribed normal heat flux at the wall becomes :

Table 3: Initial conditions of the reference case

$$
\begin{aligned}
& \begin{array}{cccc}
T(t=0) & Y_{O_{2}}(t=0) & Y_{\mathrm{CO}_{2}}(t=0) & \theta_{C}(t=0) \\
\hline 1600 \mathrm{~K} & 0.63 & 0.37 & 1
\end{array} \\
& \vec{q}_{w}^{B C} \cdot \vec{n}=-\sum_{k \in \Omega_{S}} h_{k} \dot{s}_{k}-\sum_{k \in \Omega_{G}} \Delta h_{f}^{o} \dot{s}_{k} \\
& -\rho_{w} \sum_{k \in \Omega_{G}} U_{S t e f a n} h_{s, k, w} Y_{k, w} \\
& -\frac{\dot{m}}{2} \sum_{j \in \mathcal{D}} u_{j, w}^{2}+(\overline{\bar{\tau}} \vec{u})_{w} \cdot \vec{n}
\end{aligned}
$$

The prescribed mass fraction gradient at the wall 
is given by Eq. (13). The upper face is an outlet surface that relaxes the pressure toward the targeted atmospheric pressure in a partially non-reflecting way using the NSCBC method [44, 45]. This allows to control waves reflections and avoids the propagation of non-physical fluctuations. No conditions are applied to the species mass fractions, heat flux and velocity at the outlet :

$$
\left.Y_{k}\right|_{\text {outlet }}=Y_{k}^{p},\left.\quad \vec{q}\right|_{\text {outlet }}=\vec{q}^{p}, \quad \text { and }\left.\vec{u}\right|_{\text {outlet }}=\vec{u}^{p}
$$

Where $Y_{k}^{p}, \vec{q}^{p}$ and $\vec{u}^{p}$ are respectively the values of species mass fractions, heat flux and velocity predicted by the numerical scheme. Periodic boundary patches are imposed to the lateral faces. Regarding the mesh, starting from the wall to $x / l_{\text {diff }}=0.865$, mesh cells have a size of $10 \mu \mathrm{m}$ which means that 1000 cells populate the near-wall region. For $0.865<x / l_{\text {diff }}<$ $\sqrt{D_{a}}$, a mesh coarsening has been carried out to reach a maximum cell size of $75 \mu \mathrm{m}$ at the outlet. As will be discussed in section 3.2.7, a grid convergence study is carried out to determine the proper grid resolution in the reactive near-wall region. Regarding the numerical scheme, a second-order cell-vertex implementation of the Finite Volume Lax-Wendroff convection scheme is used for time and space integration [46-49]. It is associated in this study with a Finite Element diffusion scheme with a $2 \Delta$ operator that comes from a strict application of the Finite Element Galerkin method [50]. Diffusive terms are resolved in an explicit way.

\subsubsection{Surface and gas-phase kinetic mechanisms}

The surface-phase mechanism (Table 4) was extracted from a previously developed and validated semi-detailed kinetic mechanism $[10,51,52]$. The impact of backward reactions is expected to be negligible under typical oxy-combustion conditions and the validity of this hypothesis over a wider range of conditions is outside the scope of the present study. Two additional reactions representing the adsorption of $\mathrm{CO}_{2}$ (S4) and oxygen radicals $O$ (S5) upon carbon sites have been accounted for. The kinetic parameters of surface reaction ( $\mathrm{S} 1$ to $\mathrm{S} 3$ ) related to char oxidation are similar to those recommended by Hurt et al [53], whereas those of $\mathrm{CO}_{2}$ adsorption $(\mathrm{S} 4)$ are in line with values proposed by Hecht et al [4]. The resulting detailed kinetic surface mechanism involves a lumped intermediate species, $C(O)$, standing for oxygenated surface species formed during oxidation and a single type of carbon site, $C(S)$, was chosen to describe solid carbon sites. The mechanism has been validated against continuously stirred tank reactor (CSTR) experiments by comparing predicted carbon conversion rates to the experimental data for char gasification and oxidation [54]. The simulations were carried out using Surface Chemkin kinetic solver [55]. Comparisons of the carbon conversion are shown in Fig. (6) corresponding to carbon $\mathrm{O}_{2}$ oxidation and $\mathrm{CO}_{2}$ gasification. The validation covers various concentrations of diluted $\mathrm{O}_{2}$ and $\mathrm{CO}_{2}$ using chemically inert molecular nitrogen $N_{2}$ as a balance dilution gas. Regarding the gas-phase 
mechanism, the three reversible elementary reactions of dry $C O$ oxidation given in Table 5 were extracted from the GRI 2.11 mechanism. This reaction set was then validated against shock tube experiments of Brabbs et al. for $\mathrm{CO}_{2}$ dissociation [56].

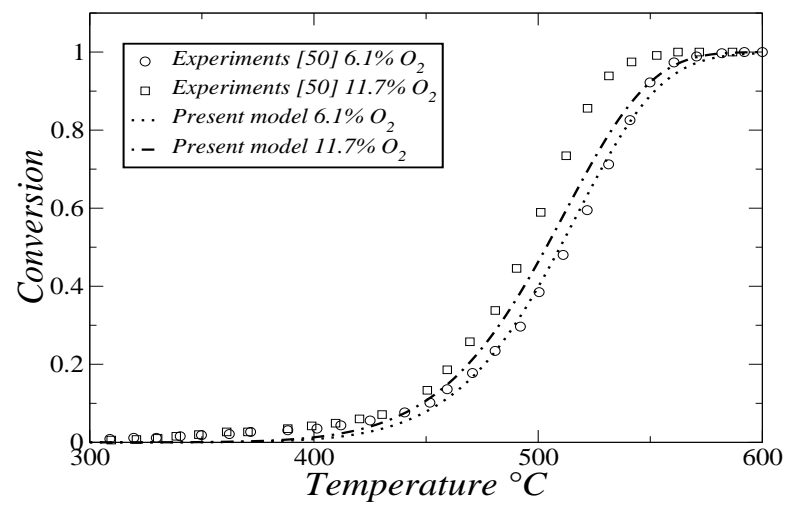

(a) $\mathrm{O}_{2} / \mathrm{N}_{2}$ environment

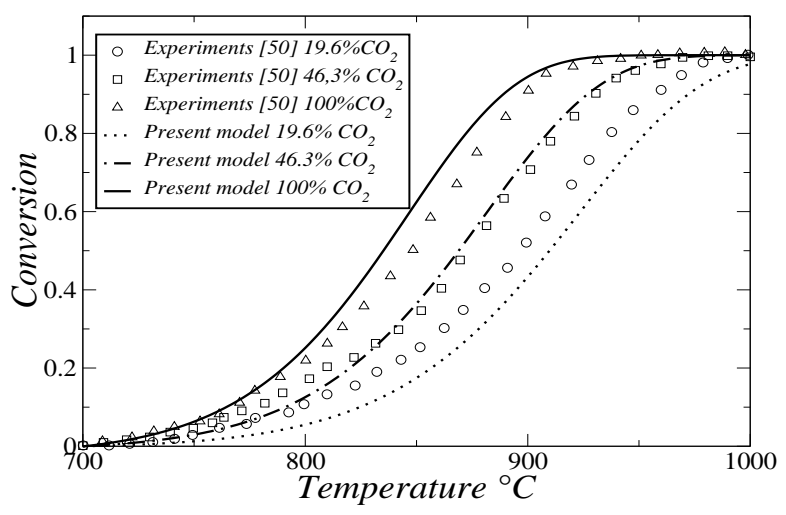

(b) $\mathrm{CO}_{2} / \mathrm{N}_{2}$ environment

Figure 6: Carbon conversion in a $\mathrm{O}_{2} / \mathrm{N}_{2}$ and $\mathrm{CO}_{2} / \mathrm{N}_{2}$ environment. The volumetric flow rate is $100 \mathrm{SCCM}$ (Standard Cubic Centimeter per Minute) and the heating rate is $10 \mathrm{~K} / \mathrm{min}$.

\subsubsection{History}

The reference case (Table 3) is characterized by two gas-phase reaction zone configurations. The first one corresponds to a wall-attached $\mathrm{CO}$-reaction zone maintained by the competition between the $\mathrm{CO} / \mathrm{O}_{2}$ combustion and surface reactions, whereas the second one is characterized by the extinction of surface reactions and a gas-phase reaction zone evolving at a certain distance of the reactive wall. Therefore, two main combustion modes characterize the reference case and the switch between these modes occurs at $t_{\text {switch }}=$ $0.22 s$ under the conditions used in this study. This time coincides with the depletion of surface carbon $\left(\theta_{c}=0\right)$ and is the same order of $\tau_{\text {chem }}$. As a consequence, time is normalized by the time corresponding to the combustion mode switch $t_{\text {switch }}$. The resulting normalized time being noted $\tau=t / t_{\text {switch }}$. A sketch of the two combustion modes is given in Fig. (7).

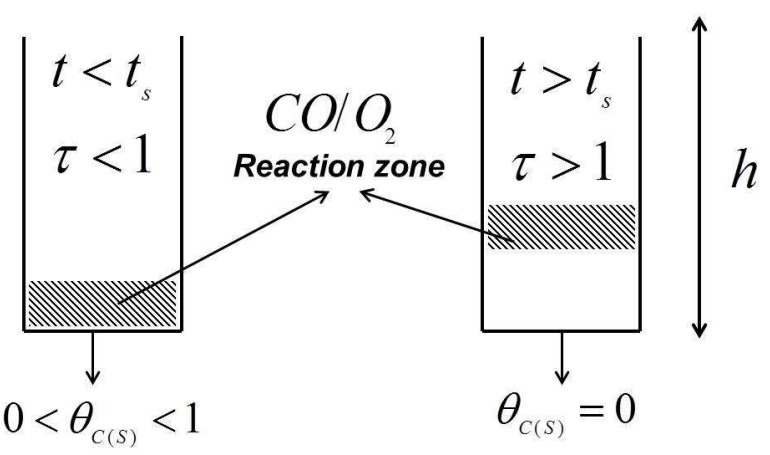

Figure 7: Gas-phase $\mathrm{CO} / \mathrm{O}_{2}$ reaction zone configurations

Fig. (8a) shows the time evolution of space averaged mass fraction of the $k^{\text {th }}$ species at the reactive 
Table 4: Surface reaction mechanism for the configuration of Fig. 5

\begin{tabular}{cccccc} 
Step & Reaction & $\alpha_{j}\left(\mathrm{~cm}^{2(n-1)} \cdot \mathrm{mol}^{1-n} \cdot \mathrm{s}^{-1}\right)$ & $\beta_{j}$ & $E_{j}\left(\mathrm{kJ.mol}^{-1}\right)$ & ref. \\
\hline S1 & $C(S)+\frac{1}{2} \mathrm{O}_{2} \rightarrow C(O)$ & $5.0 \times 10^{6}$ & 0.0 & 45 & {$[10]$} \\
S2 & $C(O) \rightarrow C O$ & $2.7 \times 10^{6}$ & 0.0 & 132 & {$[10]$} \\
S3 & $C(O)+\frac{1}{2} \mathrm{O}_{2} \rightarrow C O_{2}$ & $1.33 \times 10^{5}$ & 0.0 & 109 & {$[10]$} \\
S4 & $C O_{2}+C(S) \rightarrow C(O)+C O$ & $1.0 \times 10^{13}$ & 0.0 & 225 & {$[54]$} \\
S5 & $O+C(S) \rightarrow C(O)$ & $\left(\frac{\gamma}{\Gamma^{m}}\right) \sqrt{\frac{R}{2 \pi W_{O}}}$ & 0.5 & 0.0 & {$[57]$}
\end{tabular}

Table 5: Gas-phase reaction mechanism for the configuration of Fig. 5

\begin{tabular}{ccccc} 
Step & Reaction & $\alpha_{j}\left(\mathrm{~cm}^{3(n-1)} \cdot \mathrm{mol}^{1-n} \cdot \mathrm{s}^{-1}\right)$ & $\beta_{j}$ & $E_{j}\left(\mathrm{~kJ} . \mathrm{mol}^{-1}\right)$. \\
\hline $\mathrm{R} 1$ & $2 \mathrm{O}+\mathrm{M} \rightleftharpoons \mathrm{O}_{2}+M$ & $1.2 \times 10^{17}$ & -1.0 & 0.0 \\
$\mathrm{R} 2$ & $\mathrm{O}+\mathrm{CO}+\mathrm{M} \rightleftharpoons \mathrm{CO}_{2}+M$ & $6.02 \times 10^{14}$ & 0.0 & 12.55 \\
$\mathrm{R} 3$ & $\mathrm{O}_{2}+\mathrm{CO} \rightleftharpoons \mathrm{O}+\mathrm{CO}_{2}$ & $2.5 \times 10^{12}$ & 0.0 & 200.1
\end{tabular}

wall. These quantities are computed as :

$$
\left\langle Y_{k, \text { wall }}\right\rangle=\frac{\int_{\mathcal{S}} Y_{k}(x=0) d S}{A_{\text {wall }}}
$$

For $\tau \leq 1, \mathrm{CO}_{2}$ and $\mathrm{O}_{2}$ species present in the gas-phase diffuse to the carbonaceous wall, are adsorbed and converted on surface as shown by their respective decreasing mass fractions at the wall shown in Fig. (8a). $\mathrm{CO}_{2}$ and $\mathrm{O}_{2}$ adsorption upon the reactive surface coincides with consumption of surface carbon and $\mathrm{CO}$ production through gasification. $\mathrm{CO}$ production by the reactive surface is described by the increasing profile of $C O$ mass fraction ( Fig. (8a) ) and the decreasing profile of carbon site fraction $\theta_{C}$ ( Fig. (8b) ). $C O$ diffuses to the gas-phase and reacts with $\mathrm{O}_{2}$ thus producing $\mathrm{CO}_{2}$. Carbon monoxide consumption and carbon dioxide production through gas-phase combustion are illustrated by the respective rise and fall of $\mathrm{CO}_{2}$ and $C O$ concentrations for $0.97 \leq \tau \leq 1.0$. At $\tau=1$, surface carbon consumption reaches completion which puts an end to surface reactions. Gas-phase $\mathrm{CO}_{2} / \mathrm{O}_{2}$ combustion is the only active chemical process which gradually fades. 


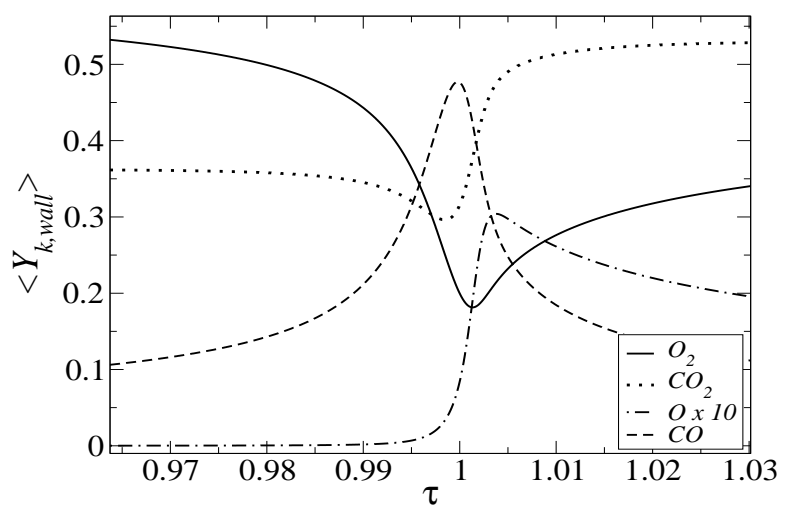

(a) Gas-phase species mass fractions at the reactive surface

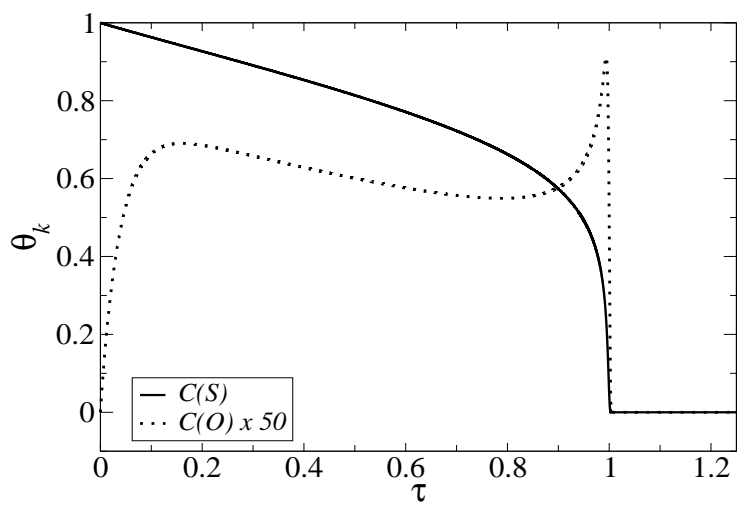

(b) Surface coverages

Figure 8: Evolution of Gas-phase species at the wall and surface coverages versus normalized time $\tau$

\subsubsection{Combustion modes}

\section{Wall-attached reaction zone :}

In order to quantify the competition between gas-phase and surface reactions, an index $I_{k}$ for each $k^{\text {th }}$ species is defined in Eq. (33):

$$
I_{k}=\frac{\left|\left\langle\dot{s}_{k}\right\rangle\right|}{\left|\left\langle\dot{s}_{k}\right\rangle\right|+\left|\left\langle\dot{\omega}_{k}\right\rangle\right|}
$$

where $\left\langle\dot{\omega}_{k}\right\rangle$ and $\left\langle\dot{s}_{k}\right\rangle$ are respectively the gas-phase and surface space averaged production/consumption rates that read:

$$
\left\langle\dot{\omega}_{k}\right\rangle=\int_{V} \dot{\omega}_{k} d V, \quad\left\langle\dot{s}_{k}\right\rangle=\int_{S} \dot{s}_{k} d S
$$

$I_{k}$ characterizes the contributions of surface and gas-phase reaction regardless of its sign (production/consumption). Fig. (9) presents the evolution of $I_{\mathrm{O}_{2}}, I_{\mathrm{CO}_{2}}$ and $I_{\mathrm{CO}}$ versus the normalized time $\tau$. As can be noticed, the index $I_{k}$ varies between 0 and 1 thus showing that the existence of a wall-attached reaction zone coincides with the competition between surface and gas-phase chemistry. As a matter of fact, $I_{O_{2}}, I_{C_{2}}$ and $I_{C O}$ are higher than 0.5 for $\tau \leq 0.24,0.7$ and 0.75 respectively, which means that surface-phase chemistry is as much involved into species consumption or production as the gas-phase one. $\mathrm{I}_{\mathrm{CO}}$ and $\mathrm{I}_{\mathrm{CO}_{2}}$ show a stiff decrease when $\tau$ tends to 1 because of the quick drop of surface carbon mass ( Fig. (8) ) putting an end to surface chemistry. This is due to the thermal runaway caused by the exothermic surface carbon oxidation (S1 to S3) and gas-phase reactions. A slight increase can be noticed before $I_{\mathrm{CO}_{2}}$ decreases rapidly which is due to the production of $\mathrm{CO}_{2}$ by reaction $S 3$ (Table 4).

$\mathrm{CO}$ provided by the surface feeds a $\mathrm{CO} / \mathrm{O}_{2}$-combustion characterized by a reaction front at proximity of the reactive wall. Fig. (10) presents the reaction zone identified by the gas-phase temperature (a) and the peaks of heat release (b). These peaks intensify and move slightly upward toward rich zones that are less $\mathrm{O}_{2}$ diffusion limited but do not go beyond $x / l_{\text {diff }}=$ 0.215 . Note that the reaction zone thickness (non-zero 


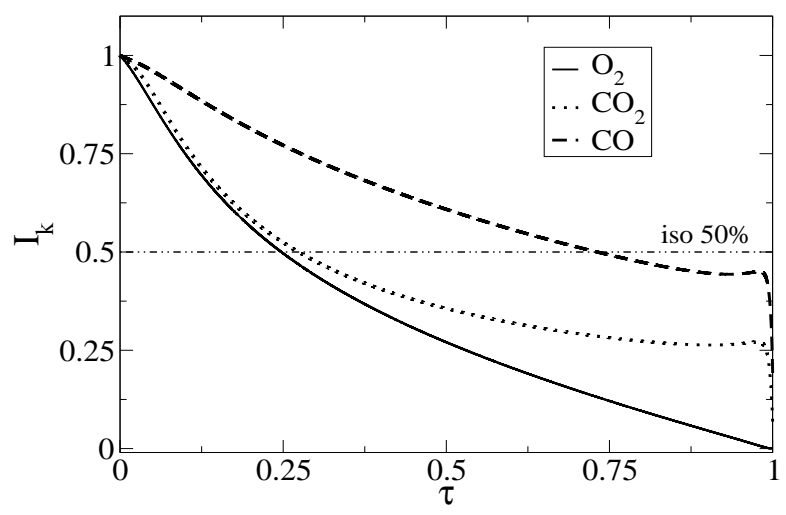

Figure 9: $I_{k}$ versus $\tau$

values of $\dot{\omega}_{T}$ ) corresponds to regions where surface produced $\mathrm{CO}$ has diffused enough to react with $\mathrm{O}_{2}$ thus covering a length that is the same order of the a priori-estimated characteristic diffusive length $l_{\text {diff }}$ as $\tau$ tends to unity, i.e, as physical time $t$ tends to one chemical characteristic time $\tau_{\text {chem }}$. This $\mathrm{CO} / \mathrm{O}_{2}$-reaction zone configuration is analogous to the "attached CO-flame" mode described by Makino [58]. The reaction zone is continuously fed by $C O$ provided by the surface $\left(\left\langle\dot{s}_{C O}\right\rangle>0\right)$, it remains attached to the wall despite the increase of $O_{2}$ gradient.

Fig. (11) shows at two different times during the wall-attached reaction zone phase the evolution of normalized species spatial profiles. Normalized scalars noted $\widehat{\Phi}_{k}$ are defined as follows :

$$
\widehat{\Phi}_{k}=\frac{\Phi_{k}-\Phi_{k, \text { min }}}{\Phi_{k, \text { max }}-\Phi_{k, \text { min }}}
$$

where $\Phi_{k, \min }$ and $\Phi_{k, \max }$ are respectively the minimum and maximum values of the scalar $\Phi_{k}$ at a given time $\tau$. $\widehat{\Phi}_{k}$ equals zero when $\widehat{\Phi}_{k}=\Phi_{k, \min }$ and 1 if $\widehat{\Phi}_{k}=\Phi_{k, \max }$.

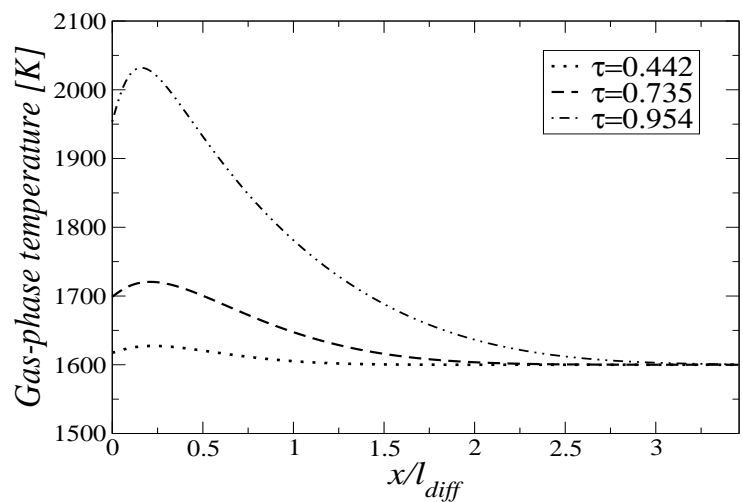

(a) Gas-phase temperature profiles

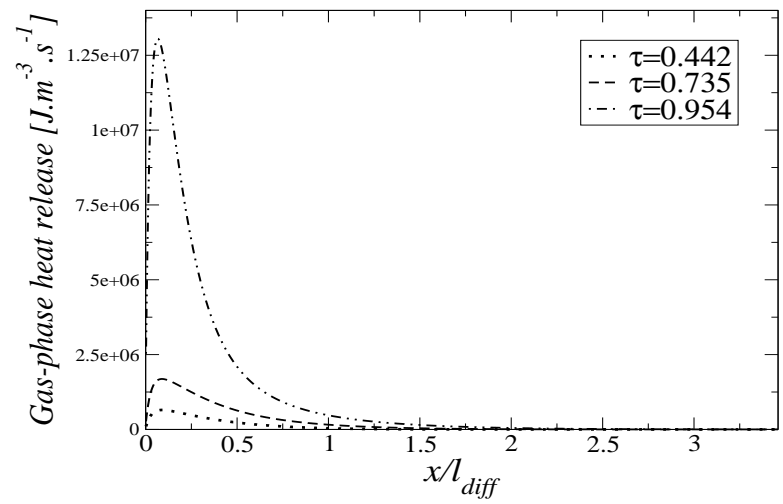

(b) Gas-phase heat release profiles

Figure 10: Temperature and gas-phase heat release profiles during the "attached CO-flame" mode 


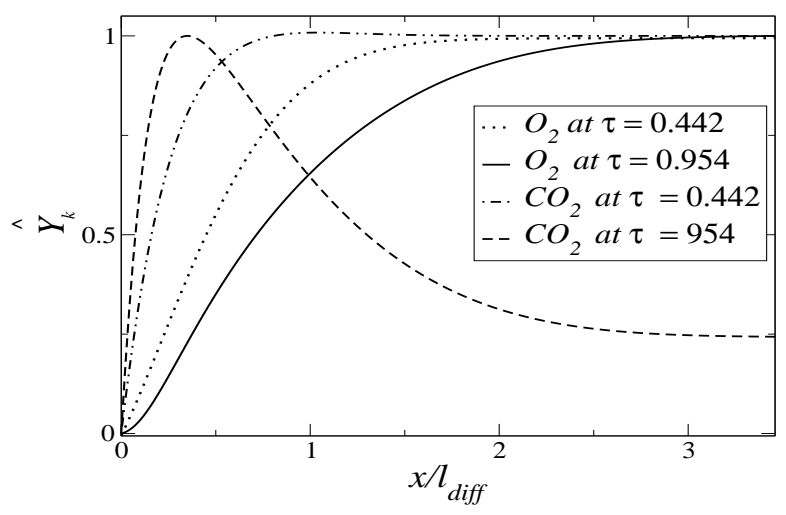

Figure 11: Spatial profiles of $\widehat{Y}_{O_{2}}$ and $\widehat{Y}_{\mathrm{CO}_{2}}$

Gas-phase combustion occurence coincides with a decrease of $\mathrm{O}_{2}$ in the reaction zone and the formation of a peak on $\mathrm{CO}_{2}$ profile. At $\tau=0.442$, the minimum of $\mathrm{CO}_{2}$ concentration is at the wall because of its consumption by surface carbon. As $\mathrm{CO} / \mathrm{O}_{2}$ reaction becomes significantly active in the gas-phase, $\mathrm{CO}_{2}$ production contributes to generate a peak in the corresponding concentration spatial profile at $x / l_{\text {diff }}=$ 0.425 at $\tau=0.954$ identifying the gas-phase reaction front. The wall-attached reaction phase $(\tau \leq 0.06)$ begins with an endothermic process during which $\mathrm{CO}_{2}$ oxidizes carbon. $\mathrm{CO}_{2}$ adsorption through surface reaction causes a drop in both wall ( Fig. (12a)) and gas-phase temperatures ( Fig. (12b) ). Indeed, the temperature decrease is due to the endothermicity of $\mathrm{CO}_{2}$ gasification of carbon. Fig. (12a) shows the decrease of the surface temperature during the endothermic phase $(\tau \leq 0.06)$ causing an increase of the positive gas-phase temperature gradient at the reactive wall as described in Fig. (12b). Meanwhile, $C O$ mass fraction gradually increases at the wall, sharpening its negative gradient as can be seen in Fig. (13).

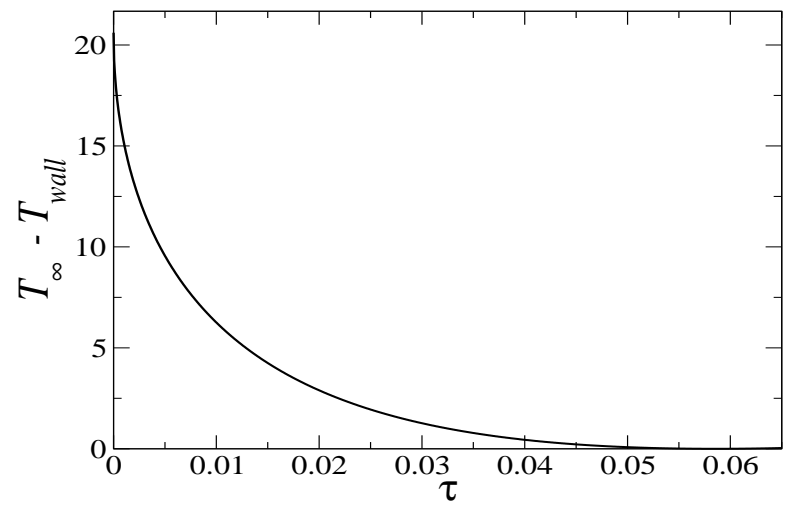

(a) Evolution of the wall temperature versus normalized time $\tau$

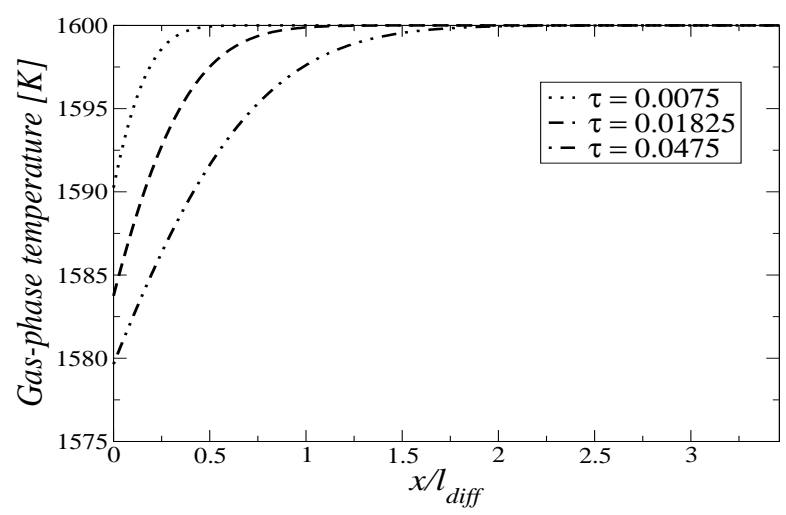

(b) Gas-phase temperature profiles

Figure 12: Evolution of the wall and gas-phase temperature profile during the endothermic phase 


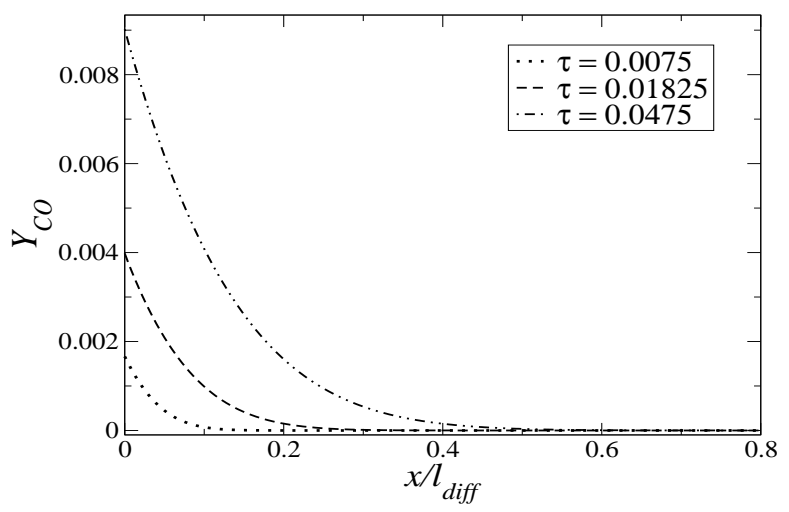

Figure 13: Gas-phase $C O$ profiles during endothermic phase

\section{Gas-phase reactions after surface carbon depletion :}

An overview of the gas-phase reaction zone displacement is shown in Figs. (14a, 14b). As carbon reaches depletion, surface reaction rates decrease which induces a change in the reaction structure. At $\tau=1.0$, surface chemistry is not active anymore $\left(\left\langle\dot{s}_{k}\right\rangle=0\right)$ as shown by Fig. (9). Temperature in proximity of the wall reaches values higher than $3000 K$ ( Fig. (14) ) favoring $\mathrm{CO}_{2}$ gas-phase dissociation thus causing a detachment of the reaction front. Fig. (14a) shows negative heat release rates near the wall region related to $\mathrm{O}_{2}$ and $\mathrm{CO}_{2}$ dissociation by $\mathrm{R} 1$ and $\mathrm{R} 2$ (Table 5). $\mathrm{CO}$ is not generated anymore by the reactive surface so that the reaction zone loses its attachment to the surface. The resulting reaction zone turns into a reaction front moving along the channel height. Fig. (14b) shows spatial profiles of the gas-phase temperature for different times during the phase where the reaction zone is detached from the wall. Unlike gas-phase temperature profile in Fig. (10a) that presents a peak, temperature profiles have a maximum at the wall resulting from the high temperature history of the phase during which the reaction zone was attached to the reactive wall. The heat-release is not high enough to induce a peak in the temperature profile.

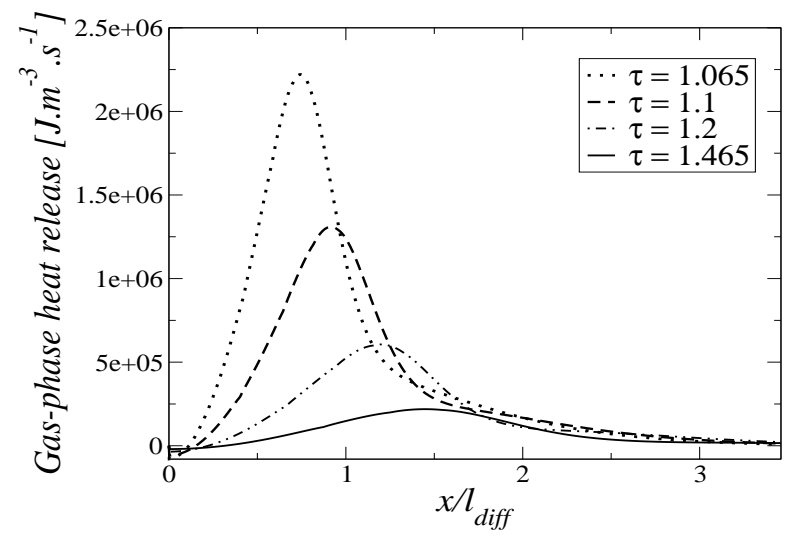

(a) Gas-phase heat release profiles

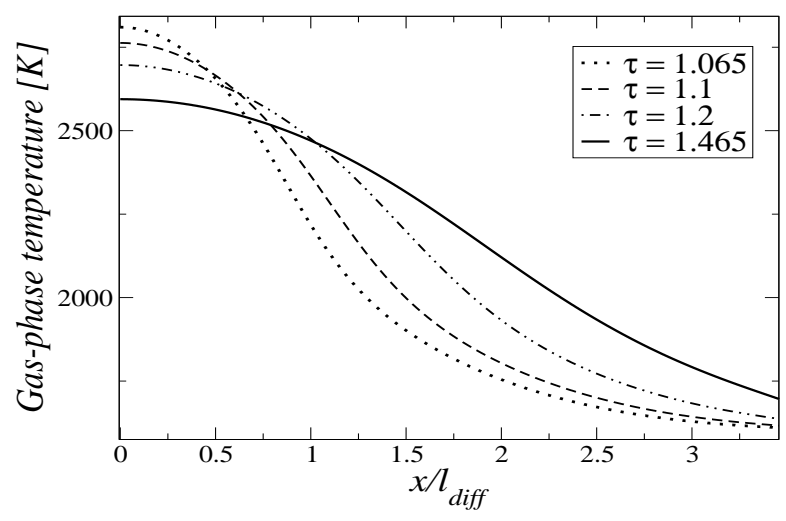

(b) Gas-phase temperature profiles

Figure 14: Gas-phase heat release and temperature profiles after surface carbon depletion

As shown in Fig. (14a), the heat release peak moves upward and decreases in amplitude with time. Indeed, 
at $\tau>1.0, C O$ diffuses in the whole domain and mixes with $\mathrm{O}_{2}$ thus creating a partially premixed reactant field where the reaction zone propagates toward $\mathrm{O}_{2}$-rich regions. The magnitude and the displacement velocity of the heat release profiles peak drop because the further the flame gets from the reactive wall, the less conducive are the conditions to sustain the gas-phase combustion. As described in Fig. (15), $C O$ concentration decreases as the distance to the wall increases, while rich $\mathrm{O}_{2}$ regions do not compensate for $C O$ diffusion limitation. The zones where $\dot{\omega}_{T}(x) \leq 0$ for a distance between 0 and $x / l_{\text {diff }} \approx$ 2.5 ( Fig. (14a) ) result from $\mathrm{O}_{2}$ dissociation into $O$ radicals and $\mathrm{CO}_{2}$ dissociation into $\mathrm{CO}$ and $\mathrm{O}$ described respectively by $R 1$ and $R 2$ given in Table 5 .

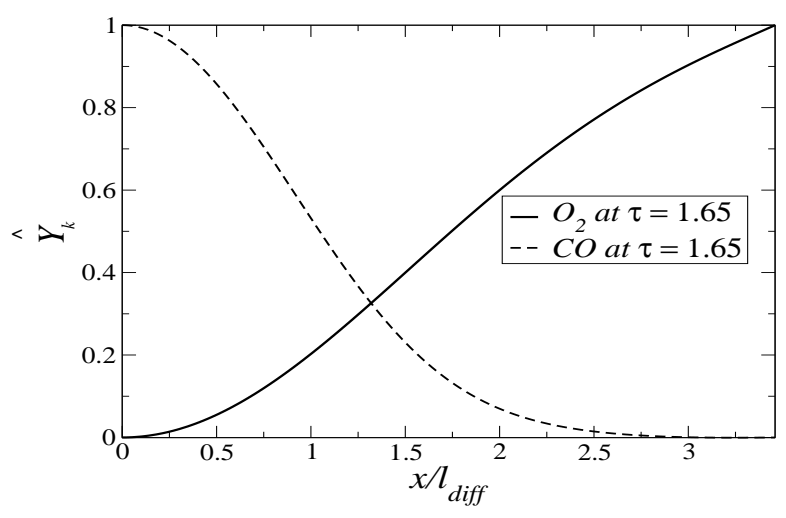

Figure 15: Spatial profiles of $\widehat{Y}_{O_{2}}$ and $\widehat{Y}_{C O}$

According to the DNS results, heterogeneous carbon ignition through $\mathrm{CO}_{2}$ and $\mathrm{O}_{2}$ adsorption can be considered as instantaneous due to the high temperature and $\mathrm{O}_{2}$ concentration. Under these extreme conditions, the resulting $C O$ production and diffusion contribute to trigger the gas-phase combustion with $\mathrm{O}_{2}$. During the competition between the heterogeneous and homogeneous reaction, the reaction zone is adjacent to the reactive wall. Carbon depletion leads to a propagation of the reaction front with a decreasing intensity toward areas that undergo less reactant depletion.

\subsubsection{A priori mass and energy fluxes evaluation}

In the case of multi-component flows, the diffusive velocity flux $\vec{J}_{k}$ can be decomposed into several terms that consist in mole fraction, pressure and temperature (Soret) gradients, whereas the heat flux $\vec{q}$ includes temperature gradient, enthalpy transport through species diffusion and the Dufour term. In our code, only mole fraction gradients are considered for the diffusive velocity flux through the Hirschfelder \& Curtiss while the Dufour term is neglected in the heat flux. Therefore, the impact of the Hirschfelder \& Curtiss approximation for the multicomponent diffusion [34, 35] and the importance of the Dufour and Soret terms are assessed by post-processing the DNS data thanks to a complex transport library ( EGlib library [43] ) :

$$
\begin{aligned}
\vec{V}_{k}^{E G l i b} & =\underbrace{\sum_{j \in \Omega_{G}} D_{k, j}^{E G l i b}\left(\vec{\nabla} X_{j}+\left(X_{j}-Y_{j}\right) \frac{\vec{\nabla} p}{p}\right)}_{\text {Diffusion velocity due to species and pressure gradients }} \\
& +\underbrace{\sum_{j \in \Omega_{G}} D_{k, j}^{E G l i b}\left(\chi_{j}^{E G l i b} \frac{\vec{\nabla} T}{T}\right)}_{\text {Soret term }}
\end{aligned}
$$




$$
\begin{aligned}
\vec{q}^{E G l i b} & =\underbrace{-\lambda^{E G l i b} \vec{\nabla} T}_{\text {Fourier term }}+\underbrace{\rho \sum_{k \in \Omega_{G}} h_{s, k} Y_{k} \vec{V}_{k}^{E G l i b}}_{\text {sensible enthalpy diffusion }} \\
& +\underbrace{p \sum_{k \in \Omega_{G}} \chi_{k}^{E G l i b} \vec{V}_{k}^{E G l i b}}_{\text {Dufour term }}
\end{aligned}
$$

$D_{k, j}^{E G l i b}$ represents the binary diffusion matrix, $\chi_{j}^{E G l i b}$ correspond to the thermal diffusion ratios and $\lambda^{E G l i b}$ refers to the a priori assessed thermal conductivity coefficient. Table 6 and Table 7 summarize the differences between the modeling of our present simulation and the a priori assessed terms for heat and mass diffusion. Since the studied case is 1D, only components in the $x$ direction (Fig. (5) ) are plotted. Fig. (16) shows the comparisons of the spatial profiles of the diffusion mass flux (Soret effect not included) and the Soret diffusion term for each gas-phase species during both reaction phases, i.e, wall-attached reaction zone phase and gas-phase reactions phase respectively at $\tau=0.65$ and $\tau=$ 1.65. Negative mass fluxes tend to compensate for low concentrations by diffusing toward the wall, whereas positive fluxes are due to species diffusing toward the outlet. Note that for all species the present mass diffusion model based on Hirschfelder \& Curtiss approximation is always understimated compared to the a priori EGLIB based mass diffusion. The pressure gradient being negligible in our case, this overestimation can be attributed to the hypothesis of constant Schmidt numbers used by our code to estimate non-binary mass diffusion coefficients. The relative error is around 5 percent which is fairly acceptable. Concerning the Soret effect which is not taken into account by our model, a priori calculations show that for $\mathrm{CO}_{2}$ and $\mathrm{O}_{2}$ species, the corresponding term is very small compared to mass diffusion in our case as shown by Figs. (16a, 16b, 16c, 16d) while it is negligible for $C O$ species as given in Figs. (16e, 16f). As a result, species concentration gradients play in our case a much more important role for species diffusion than the temperature gradient.

Heat fluxes linked to Fourier, sensible enthalpy diffusion and Dufour diffusion are also calculated a priori thanks to the EGlib library fed by the DNS data obtained under the Hirschfelder \& Curtiss approximation. The estimation of the heat flux given by the EGLIB library enables to estimate the error related to the Hirschfelder \& Curtiss approximation for species diffusion, moreover, the assumption of constant Prandtl numbers is made in our code to calculate the thermal conduction coefficient $\lambda$ appearing in the Fourier diffusion term while the Dufour effect is not taken into account during the calculations. Hence, this section aims at verifying its importance.

Fig. (17) compare the spatial profiles of sensible enthalpy diffusion, Fourier and Dufour diffusion for both reaction phases, i.e, wall-attached reaction phase and gas-phase reaction phase at $\tau=0.65$ and $\tau=$ 1.65. Regarding the sensible enthalpy transport through species diffusion, our DNS logically overestimates it since it overpredicts already the mass diffusion flux. The overestimation of the Fourier diffusion heat flux can be 
Table 6: Diffusion mass flux modeling : Present simulation versus a priori assessment

\begin{tabular}{ccc} 
Model & $\vec{\nabla} X_{k}, \vec{\nabla} p$ & Soret \\
\hline Present simulation & Hirschfelder \& Curtiss approximation & Neglected \\
a priori assessment & $\sum_{j \in \Omega_{G}} D_{k, j}^{E G l i b}\left(\vec{\nabla} X_{j}+\left(X_{j}-Y_{j}\right) \frac{\vec{\nabla} p}{p}\right)$ & $\sum_{j \in \Omega_{G}} D_{k, j}^{E G l i b}\left(\chi_{j}^{E G l i b} \frac{\vec{\nabla} T}{T}\right)$
\end{tabular}

Table 7: Heat flux modeling : Present simulation versus a priori assessment

\begin{tabular}{cccc} 
Model & Fourier & Sensible enthalpy diffusion & Dufour \\
\hline Present simulation & $-\lambda \vec{\nabla} T$ & $\rho \sum_{k \in \Omega_{G}} h_{s, k} Y_{k} \vec{V}_{k}$ & Neglected \\
a priori assessment & $-\lambda^{E G l i b} \vec{\nabla} T$ & $\rho \sum_{k \in \Omega_{G}} h_{s, k} Y_{k} \vec{V}_{k}^{E G l i b}$ & $p \sum_{k \in \Omega_{G}} \chi_{k}^{E G l i b} \vec{V}_{k}^{E G l i b}$
\end{tabular}

attributed to the hypothesis of constant Prandtl number used by our code to estimate thermal conductivity coefficient. Though, the relative error is around 6.5 percent which is satisfying. This analysis points out also that the Fourier and sensible enthalpy diffusion are of the same order of magnitude. Concerning the Dufour effect, which is not taken into account in our code, a posteriori calculations show in our case that it is negligible as given in Fig. (17). The system energy is thus much more sensitive to temperature gradients and species enthalpy diffusion than species diffusion.

\subsubsection{Parametric study}

A parametric study is carried out by varying initial $\mathrm{O}_{2}$ and $\mathrm{CO}_{2}$ concentrations. In order to characterize the effect of the initial ratio $\left.\left(X_{O_{2}} / X_{\mathrm{CO}_{2}}\right) \quad\right|_{t=0}$ on the combustion mode switch. Moreover, the influence on surface and gas-phase chemistry competition, i.e, $I_{k}(\tau)=0.5$ is studied. Fig. (18) shows the evolution of $\tau_{\text {switch }}$ and $\tau_{1 / 2, k}$ versus the molar ratio $\left.\left(X_{O_{2}} / X_{C_{2}}\right)\right|_{t=0}$ for each species.

The parameters $\tau_{\text {switch }}$ and $\tau_{1 / 2, k}$ stand respectively for the physical time corresponding to the combustion mode switch and the physical time below which $I_{k} \leqslant 0.5$ both normalized by their values for the reference case corresponding initially to $X_{O_{2}}=0.7$, i.e, $\left.\left(X_{O_{2}} / X_{C_{2}}\right)\right|_{t=0}=2.33$. As $O_{2}$ initial concentration increases, exothermic surface oxidation is enhanced which increases carbon combustion rate thus resulting in a decrease of $\tau_{\text {switch }}$ as the $\left.\left(X_{\mathrm{O}_{2}} / X_{\mathrm{CO}_{2}}\right)\right|_{t=0}$ ratio increases. $\tau_{1 / 2, O_{2}}$ then rises because richer $O_{2}$ mixtures favour surface oxidation reactions (Table 4, reactions S1 to S3) which in turn enhances the surface rate $\dot{S}_{O_{2}}$. As a consequence, the competition between $\mathrm{O}_{2}$ adsorption by surface carbon and its consumption by the gas-phase combustion is extended to a wider time range.

On the contrary, as the initial molar ratio $\left.\left(X_{O_{2}} / X_{\mathrm{CO}_{2}}\right)\right|_{t=0}$ is increased, $\tau_{1 / 2, \mathrm{CO}_{2}}$ drops because less $\mathrm{CO}_{2}$ species is available for surface gasification reaction (Table 4 


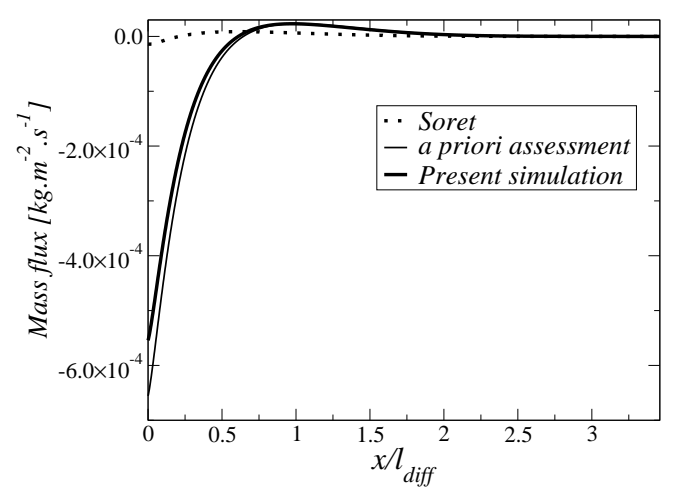

(a) Mass fluxes for $\mathrm{CO}_{2}$ species

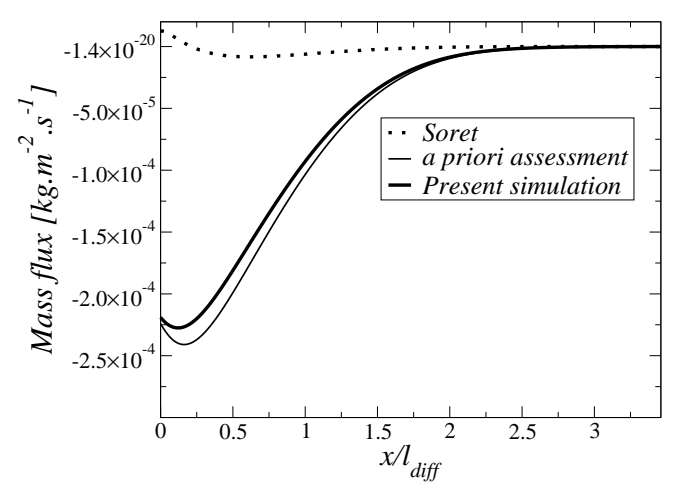

(c) Mass fluxes for $\mathrm{O}_{2}$ species

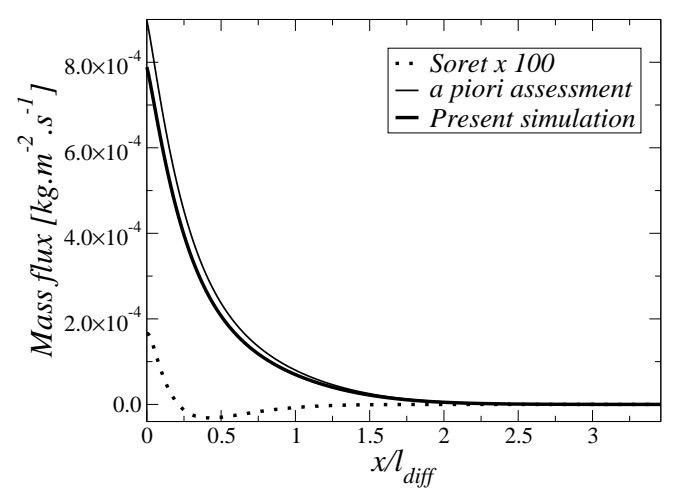

(e) Mass fluxes for $C O$ species

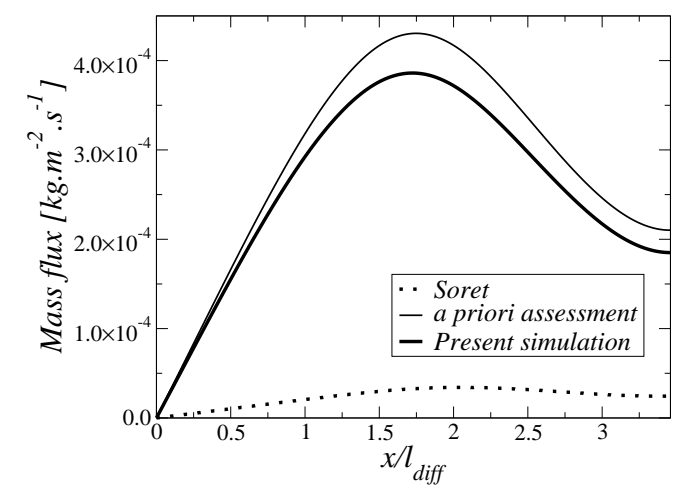

(b) Mass fluxes for $\mathrm{CO}_{2}$ species

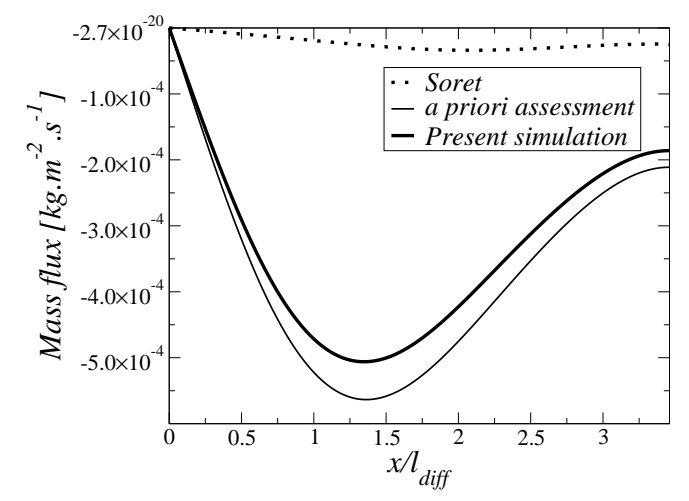

(d) Mass fluxes for $O_{2}$ species

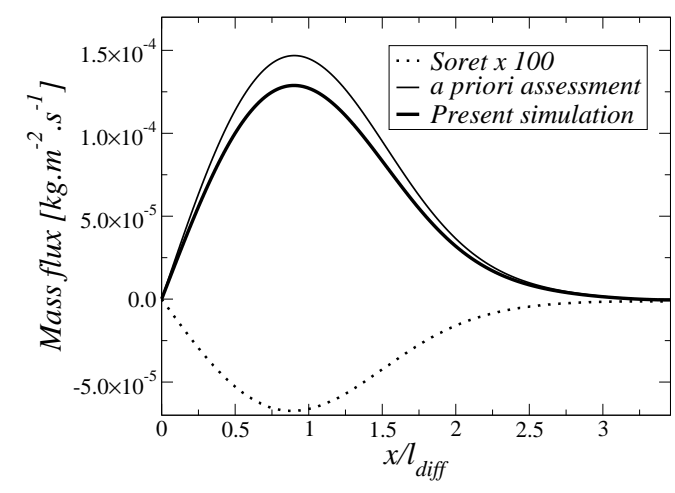

(f) Mass fluxes for $\mathrm{CO}$ species

Figure 16: Comparison of a priori assessment versus the present simulation : Soret term versus diffusion induced by molar fraction and pressure gradients at $\tau=0.65$ (left) and $\tau=1.65$ (right) 


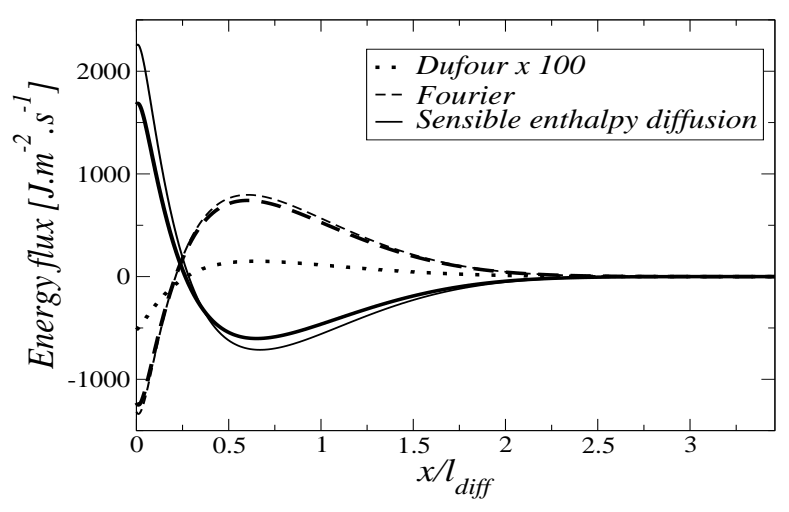

(a) Energy fluxes at $\tau=0.65$

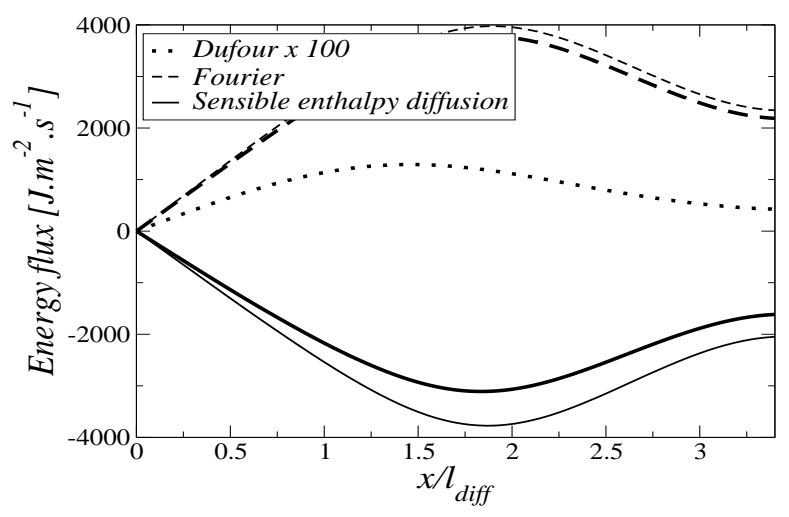

(b) Energy fluxes at $\tau=1.65$

Figure 17: Comparison of a priori assessment (thin lines) versus the present simulation (thick lines).

reactions $S 4$ ) which reduces the surface rate $\dot{\mathrm{S}}_{\mathrm{CO}_{2}}$. The resulting competition between $\mathrm{CO}_{2}$ consumption by the reactive surface and its production through gas-phase combustion becomes shorter.

\subsubsection{Grid convergence}

A global criterion was written in order to estimate the proper grid resolution in the near-wall region. The criterion gave a cell height of $h_{\min }=15 \mu \mathrm{m}$ and was established according to the following equality :

$d t_{\text {schem,min }} \approx d t_{F o, \text { min }}$. With $d t_{F o, \text { min }}=F o\left(h_{\text {min }}^{2} / v\right)$.

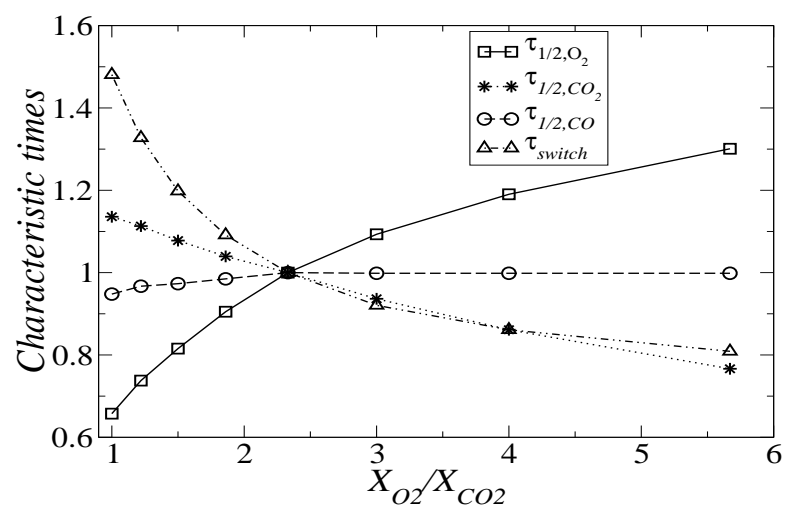

Figure 18: Characteristic times versus initial molar ratio $\left.\left(X_{\mathrm{O}_{2}} / X_{\mathrm{CO}_{2}}\right)\right|_{t=0}$. The case $\left.\left(X_{\mathrm{O}_{2}} / X_{\mathrm{CO}_{2}}\right)\right|_{t=0}=2.33$ is taken as a reference

The resulting minimum cell height is $h_{\min }=$ $\sqrt{(v / F o) d t_{\text {schem,min }}} \cdot d t_{\text {schem }}=\min \left(\frac{\Gamma W_{k}}{\dot{s}_{k}}\right)$ corresponds to the minimum surface chemistry time step estimated thanks to homogeneous calculations and $d t_{F o, \min }$ is an $a$ priori estimation of the diffusive minimum time step which is the limiting time step in our case. A constant grid resolution was applied for the diffusive length with a cell height corresponding to $h_{\min }$ and a mesh coarsening has been carried out between $x / l_{\text {diff }}=0.865$ and the outlet. Nevertheless, a grid convergence was necessary to determine properly the mesh size. The grid convergence was performed for 4 different meshes by solely varying the diffusive length mesh size between $h_{\min }=15 \mu m$ and $h_{\min }=5 \mu m$. Fig. (19) shows near-wall gradients of $\mathrm{CO}_{2}$ species mass fractions and gas-phase temperature at a time where gradients are strongest, i.e, at $\tau=0.955$. It shows that the grid convergence was reached at $7 \mu m$. The relative differences between the $7 \mu m$ grid resolution and the present mesh of $10 \mu m$ are 


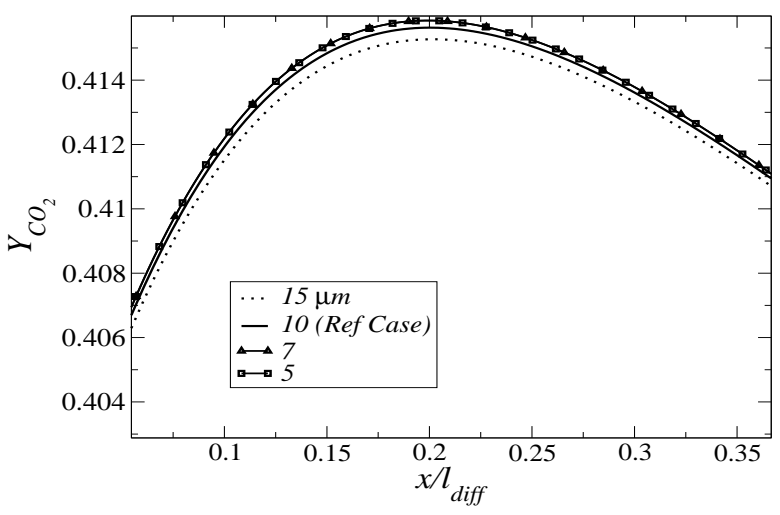

(a) Gas-phase $\mathrm{CO}_{2}$ species mass fraction profiles

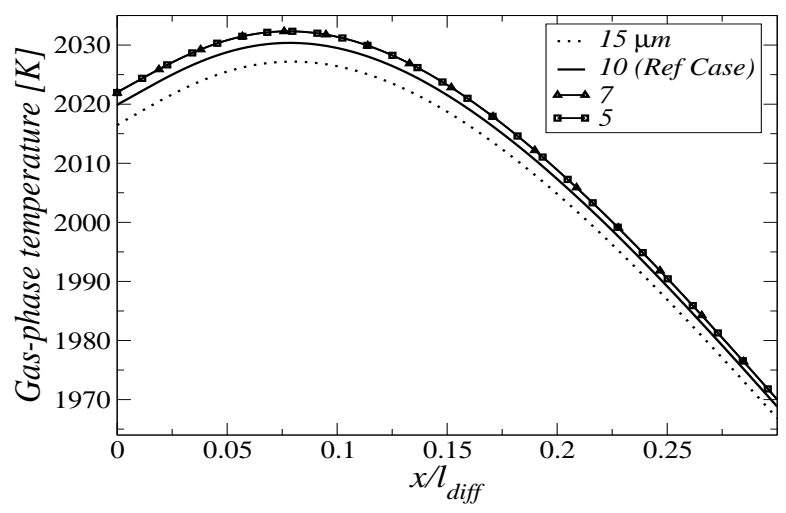

(b) Gas-phase temperature profiles

Figure 19: Grid convergence for four different near wall resolutions at $\tau=0.955$

around $0.1 \%$ only. Therefore, the $10 \mu m$ resolution was chosen because it is judged fine enough to resolve the stiffest gradients at the reactive wall and the gas-phase reaction zone thickness with reasonable numerical limiting time steps.

\section{Conclusions}

A boundary condition for the impact of surface reactions on the heat flux at the reactive wall has been developed and applied to Direct Numerical Simulations. An original analytical solution has thus been derived for a flow bounded by two reactive surfaces. A special attention has been given to the numerical setup so that compatibility with a steady-state is achieved. This approach enables to compare analytical solutions with stationary numerical predictions thus validating numerical species boundary condition for the heterogeneous surface reactions.

DNS accounting for multi-species diffusion and micro-kinetics taking into account the Stefan flux were subsequently performed to study carbonaceous wall combustion under oxy-fuel conditions. Transition from endothermic gasification to surface carbon oxidation and gas-phase ignition were evidenced thanks to the analysis of the time-dependent surface and gas-phase species production rates and transient histories of surface species concentrations as well as spatial profiles of gas-phase species, heat release and temperature. The reference case that consists in a quiscient mixture containing 70 percent of $\mathrm{O}_{2}$ and 30 percent of $\mathrm{CO}_{2}$ in volume is characterized by two gas-phase reaction zone configurations. The first one corresponds to a wall-adjacent $\mathrm{CO}$-reaction zone maintained by the competition between the $\mathrm{CO} / \mathrm{O}_{2}$ combustion and surface reactions. $C O$ surface desorption contributes to the continous feeding of the reaction zone which in turn produces $\mathrm{CO}_{2}$ that diffuses to the surface and adsorbs on surface carbon thus providing an additional $C O$ formation pathway. Once carbon has been totally consumed the reaction zone moves toward the outlet 
while its intensity is progressively dumped. Therefore, there is a strong interaction between the surface and gas-phase kinetics depending on the chemical conditions. Note that this reaction zone occuring at a certain distance of the reactive wall is observed despite the absence of volatiles in the gas-phase.

The impact of the Hirschfelder \& Curtiss approximation for the multicomponent diffusion and the importance of the Dufour and Soret terms were assessed by post-processing the DNS data thanks to a complex transport library (EGlib library). A priori post-processing of the DNS showed that species concentration gradients have a much more important contribution to the diffusion velocity than the temperature gradient (Soret effect) whereas the system energy is much more sensitive to temperature gradients (Fourier) and sensible enthalpy diffusion that are of the same order of magnitude. The Dufour effect being also negligible, the Hirschfelder \& Curtiss approximation is satisfying since the relative error between the fluxes predicted by our DNS and the ones estimated by the EGLIB library is around 6 percent. The discrepancies can be attributed to the hypothesis of constant Schmidt and Prandtl numbers used to estimate non-binary mass diffusion coefficients and thermal conduction coefficient respectively.

Eventually, the effect of initial concentrations of $\mathrm{O}_{2}$ and $\mathrm{CO}_{2}$ on characteristic times of gas and surface reactions and times corresponding to the switch between the two gas-phase reactions structures has been assessed. As the $\left.\left(X_{\mathrm{O}_{2}} / X_{\mathrm{CO}_{2}}\right)\right|_{t=0}$ ratio increases, the switch between the two modes of gas-phase reactions occurs earlier and the competition between $\mathrm{CO}_{2}$ consumption by the reactive surface and its production through gas-phase combustion becomes shorter. In contrast, the surface consumption of $\mathrm{O}_{2}$ was seen to compete gas-phase combustion for a wider time range.

\section{Acknowledgement}

The authors thank Dr. Guillaume Moula for his contributions to the analytical solution derivation for the species boundary condition validation. 


\section{References}

[1] J. Bouillard, A. Vignes, O. Dufaud, L. Perrin, D. Thomas, Ignition and explosion risks of nanopowders, Journal of Hazardous Materials 181 (1-3) (2010) 873 - 880.

[2] E. S. Hecht, Single particle studies of pulverized coal oxy-combustion, Ph.D. thesis, The University of Utah (2013).

[3] D. Kim, S. Choi, C. R. Shaddix, M. Geier, Effect of $\mathrm{CO} 2$ gasification reaction on char particle combustion in oxy-fuel conditions, Fuel 120 (2014) 130 - 140.

[4] E. S. Hecht, C. R. Shaddix, M. Geier, A. Molina, B. S. Haynes, Effect of $\mathrm{CO} 2$ and steam gasification reactions on the oxy-combustion of pulverized coal char, Combustion and Flame 159 (11) (2012) 3437 - 3447

[5] S. Granata, T. Faravelli, E. Ranzi, A wide range kinetic modeling study of the pyrolysis and combustion of naphthenes, Combustion and Flame 132 (3) (2003) 533 - 544.

[6] T. F. Wall, G.-s. Liu, H.-w. Wu, D. G. Roberts, K. E. Benfell, S. Gupta, J. A. Lucas, D. J. Harris, The effects of pressure on coal reactions during pulverised coal combustion and gasification, Progress in Energy and Combustion Science 28 (5) (2002) 405-433.

[7] L. Chen, S. Z. Yong, A. F. Ghoniem, Oxy-fuel combustion of pulverized coal: Characterization, fundamentals, stabilization and CFD modeling, Progress in Energy and Combustion Science 38 (2) (2012) $156-214$

[8] P. A. Libby, T. R. Blake, Theoretical study of burning carbon particles, Combustion and Flame 36 (1979) 139 - 169.

[9] G. Huang, F. J. Vastola, A. W. Scaroni, Temperature gradients in the gas phase surrounding pyrolyzing and burning coal particles, Energy \& fuels 2 (4) (1988) 385-390.

[10] K. Leistner, A. Nicolle, D. Berthout, P. da Costa, Kinetic modelling of the oxidation of a wide range of carbon materials, Combustion and Flame 159 (1) (2012) 64 - 76.

[11] R. H. Essenhigh, M. K. Misra, D. W. Shaw, Ignition of coal particles: A review, Combustion and Flame 77 (1) (1989) 3 30.

[12] A. Makino, C. K. Law, An analysis of the transient combustion and burnout time of carbon particles, Proceedings of the
Combustion Institute 32 (2) (2009) 2067 - 2074.

[13] D. Spalding, The prediction of mass transfer rates when equilibrium does not prevail at the phase interface, International Journal of Heat and Mass Transfer 2 (4) (1961) 283 - 313.

[14] A. Makino, C. Law, Ignition and extinction of co flame over a carbon rod, Combustion Science and Technology 73 (4-6) (1990) 589-615.

[15] C. Zou, L. Cai, C. Zheng, Numerical research on the homogeneous/heterogeneous ignition process of pulverized coal in oxy-fuel combustion, International Journal of Heat and Mass Transfer 73 (2014) $207-216$

[16] E. S. Hecht, C. R. Shaddix, J. S. Lighty, Analysis of the errors associated with typical pulverized coal char combustion modeling assumptions for oxy-fuel combustion, Combustion and Flame 160 (8) (2013) 1499-1509.

[17] M. Zhang, J. Yu, X. Xu, A new flame sheet model to reflect the influence of the oxidation of $\mathrm{CO}$ on the combustion of a carbon particle, Combustion and Flame 143 (3) (2005) 150 - 158

[18] R. Lewtak, A. Milewska, Application of different diffusion approaches in oxy-fuel combustion of single coal char particles, Fuel 113 (2013) $844-853$.

[19] O. Deutschmann, R. Schmidt, F. Behrendt, J. Warnatz, Numerical modeling of catalytic ignition, in: Symposium (International) on Combustion, Vol. 26, Elsevier, 1996, pp. 1747-1754.

[20] L. L. Raja, R. J. Kee, L. R. Petzold, Simulation of the transient, compressible, gas-dynamic behavior of catalytic-combustion ignition in stagnation flows, Symposium (International) on Combustion 27 (2) (1998) 2249 - 2257.

[21] M. Matalon, Complete burning and extinction of a carbon particle in an oxidizing atmosphere, Combustion Science and Technology 24 (3-4) (1980) 115-127.

[22] J. C. Lee, R. A. Yetter, F. L. Dryer, Transient numerical modeling of carbon particle ignition and oxidation, Combustion and Flame 101 (4) (1995) 387 - 398.

[23] P. A. Libby, T. R. Blake, Burning carbon particles in the presence of water vapor, Combustion and Flame 41 (1981) 123 $-147$. 
[24] A. Makino, C. Law, Quasi-steady and transient combustion of a carbon particle: Theory and experimental comparisons, Symposium (International) on Combustion 21 (1) (1988) 183 $-191$.

[25] V. M. Janardhanan, O. Deutschmann, Modeling diffusion limitation in solid-oxide fuel cells, Electrochimica Acta 56 (27) (2011) 9775-9782.

[26] O. Deutschmann, Interactions between transport and chemistry in catalytic reactors, Ruprecht-Karls-Universität Heidelberg, 2001.

[27] O. Deutschmann, L. Maier, U. Riedel, A. Stroemman, R. Dibble, Hydrogen assisted catalytic combustion of methane on platinum, Catalysis Today 59 (1) (2000) 141-150.

[28] H. Motz, H. Wise, in: Journal of Chemical Physics, Vol. 32, 1960.

[29] T. Schoenfeld, The AVBP handbook (2008).

[30] V. Granet, S. Menon, O. Vermorel, G. Staffelbach, T. Poinsot, A. Roux, Large-eddy simulation of a swirled lean premixed gas turbine combustor: a comparison of two compressible codes, in: 51st AIAA Aerospace Sciences Meeting including the New Horizons Forum and Aerospace Exposition, 2013.

[31] F. Cadieux, J. A. Domaradzki, T. Sayadi, S. Bose, F. Duchaine, DNS and LES of separated flows at moderate Reynolds numbers, in: Proceedings of the Summer Program, 2012, p. 77.

[32] T. Poinsot, J. Dombard, V. Moureau, N. S. G. Staffelbach, V. Bodoc, Experimental and numerical study of the influence of small geometrical modifications on the dynamics of swirling flows, in: Proceedings of the Summer Program, 2012, p. 1.

[33] H. B. Toda, O. Cabrit, K. Truffin, G. Bruneaux, F. Nicoud, Assessment of subgrid-scale models with a large-eddy simulation-dedicated experimental database: The pulsatile impinging jet in turbulent cross-flow, Physics of Fluids 26 (7) (2014) 075108 .

[34] C. F. Curtiss, R. B. Bird, Multicomponent diffusion, Industrial and Engineering Chemistry Research 38 (7) (1999) 2515-2522.

[35] J. O. Hirschfelder, C. F. Curtiss, R. B. Bird, et al., Molecular theory of gases and liquids, Vol. 26, Wiley New York, 1954.

[36] O. Cabrit, Wall modeling of the flow inside solid rocket motor nozzles, Ph.D. thesis, Montpellier 2 (2009).

[37] O. Cabrit, F. Nicoud, Direct numerical simulation of a reacting turbulent channel flow with thermochemical ablation, Journal of Turbulence 11 (44) (2010) 1-33.

[38] H. Schlichting, K. Gersten, Boundary Layer Theory, 8th Edition, Springer-Verlag, Berlin Heidelberg, Germany, 2000.

[39] J. C. Y. Koh, J. P. Hartnett, Skin friction and heat transfer for incompressible laminar flow over porous wedges with suction and variable wall temperature, Int. J. Heat Mass Transfer2 (3) (1961) 185-198.

[40] E. A. Hamza, Suction and injection effects on a similar flow between parallel plates, Journal of Physics D: Applied Physics32 (4) (1999) 656-663.

[41] D. E. Rosner, Convective diffusion as an intruder in kinetic studies of surface catalyzed reactions, AIAA Journal 2 (4) (1964) 593-610.

[42] L. Duan, M. P. Martín, Procedure to validate direct numerical simulations of wall-bounded turbulence including finite-rate reactions, AIAA Journal 47 (1) (2009) 244-251.

[43] A. Ern, V. Giovangigli, Eglib: A general-purpose fortran library for multicomponent transport property evaluation, CERMICS, ENPC and Centre de Mathématiques Appliquées, CNRS.

[44] F. N. L. Selle, T. Poinsot, Actual impedance of nonreflecting boundary conditions: Implications for computation of resonators, AIAA Journal 42 (5) (2007) 958 - 964.

[45] D. H. Rudy, J. C. Strikwerda, A nonreflecting outflow boundary condition for subsonic navier-stokes calculations, Journal of Computational Physics 36 (1) (1980) 55 - 70.

[46] P. Crumpton, J. Mackenzie, K. Morton, Cell vertex algorithms for the compressible navier-stokes equations, Journal of Computational Physics 109 (1) (1993) 1 - 15.

[47] P. Lax, B. Wendroff, Systems of conservation laws, Communications on Pure and Applied mathematics 13 (2) (1960) 217-237.

[48] P. D. Lax, B. Wendroff, Difference schemes for hyperbolic equations with high order of accuracy, Communications on pure and applied mathematics 17 (3) (1964) 381-398.

[49] V. Moureau, A. René, Simulation aux grandes échelles de 
l'aérodynamique interne des moteurs à piston, Ph.D. thesis, Châtenay-Malabry, Ecole Centrale Paris (2004).

[50] O. Colin, M. Rudgyard, Development of high-order taylor-galerkin schemes for les, Journal of Computational Physics 162 (2) (2000) 338-371.

[51] E. S. Hecht, C. R. Shaddix, A. Molina, B. S. Haynes, Effect of $\operatorname{co} 2$ gasification reaction on oxy-combustion of pulverized coal char, Proceedings of the combustion institute 33 (2) (2011) 1699-1706.

[52] L. R. Radovic, H. Jiang, A. A. Lizzio, A transient kinetics study of char gasification in carbon dioxide and oxygen, Energy \& fuels 5 (1) (1991) 68-74.

[53] R. H. Hurt, J. M. Calo, Semi-global intrinsic kinetics for char combustion modeling, Combustion and Flame 125 (3) (2001) $1138-1149$.

[54] V. Cozzani, Reactivity in oxygen and carbon dioxide of char formed in the pyrolysis of refuse-derived fuel, Industrial and Engineering Chemistry Research 39 (4) (2000) 864-872.

[55] M. E. Coltrin, R. J. Kee, F. M. Rupley, E. Meeks, Surface chemkin III: A fortran package for analyzing heterogeneous chemical kinetics at a solid-surface-gas-phase interface, Sandia National Laboratories Report SAND96-8217.

[56] T. A. Brabbs, F. E. Belles, S. A. Zlatarich, Shock-tube study of carbon dioxide dissociation rate, The Journal of Chemical Physics 38 (8) (1963) 1939-1944.

[57] G. Miessen, F. Behrendt, O. Deutschmann, J. Warnatz, Numerical studies of the heterogeneous combustion of char using detailed chemistry, Chemosphere 42 (57) (2001) 609 613 , proceedings of the 6th Intl Congress on Toxic combustion.

[58] A. Makino, An attempt for applying formulation of the carbon combustion in the stagnation flowfield to some experimental comparisons related to the boundary layer combustion, Combustion and Flame 161 (6) (2014) 1537 - 1546.

[59] A. Ern, V. Giovangigli, Multicomponent transport algorithms, Vol. 24, Springer, 1994.

[60] V. Giovangigli, Multicomponent flow modeling, Springer, 1999.

[61] K. K. Kuo, Principles of combustion, Wiley New York et al., 1986.
[62] M. E. Coltrin, H. Moffat, R. Kee, F. Rupley, Creslaf (version 4. 0): A fortran program for modeling laminar, chemically reacting, boundary-layer flow in cylindrical or planar channels, Tech. rep., Sandia National Labs., Albuquerque, NM (United States); Sandia National Labs., Livermore, CA (United States) (1993). 


\section{Appendix A.}

\section{Gas-phase governing equations}

Multi-species reactive flow equations :

A brief summary of the governing equations of the LES/DNS flow solver AVBP is provided in this section. Please, refer to the AVBP handbook [29] and http://www.cerfacs.fr/avbp/AVBP_V6.X/HANDBOOK/ AVBP/handbook.pdf for further details. The AVBP solver uses a conservative finite-volume approach. The following description focuses on a conservative formulation of the unfiltered compressible Navier-Sokes equations used in Direct Numerical simulation (DNS). The set of equations describing the evolution of a compressible flow with gas-phase chemical reactions is written in the following vectorial way :

Momentum :

$$
\forall i \in \mathcal{D}: \frac{\partial \rho u_{i}}{\partial t}+\vec{\nabla} \cdot\left(\rho u_{i} \vec{u}\right)=\vec{\nabla} \cdot\left(\vec{\tau}_{i}-p \overrightarrow{\delta_{i}}\right)
$$

Energy:

$\frac{\partial \rho E}{\partial t}+\vec{\nabla} \cdot(\rho E \vec{u})=\dot{\omega}_{T}-\vec{\nabla} \cdot(\vec{q})+\vec{\nabla} \cdot((\overline{\bar{\tau}}-p \overline{\bar{\delta}}) \vec{u})$

Species :

$$
\forall k \in \Omega_{G}: \frac{\partial \rho Y_{k}}{\partial t}+\vec{\nabla} \cdot\left(\rho(\vec{u}+\vec{V}) Y_{k}\right)=\dot{\omega}_{k}
$$

$E$ is the total non-chemical energy defined as follows :

$$
E=\sum_{k \in \Omega_{G}} h_{s, k} Y_{k}-(p / \rho)+1 / 2 \sum_{j \in \mathcal{D}} u_{j}^{2}
$$

While $\rho$ is given by :

$$
\rho=\sum_{k \in \Omega_{G}} \rho_{k}=\sum_{k \in \Omega_{G}} \rho Y_{k}
$$

$\rho E$ is transported and the temperature values are tabulated thanks to sensible energy look-up table. The pressure $p$ is given by the equation of state for ideal gas mixture.

Modeling diffusion of species, heat and momentum :

The diffusion velocities $V_{k, i}$ are obtained thanks to the Hirschfelder \& Curtiss approximation [35]. A correction velocity is introduced so that the compatibility between species and mass conservation is ensured. The heat flux vector $\vec{q}$ stands for heat conduction (Fourier term) and heat transport by species diffusion:

$$
\vec{q}=-\lambda^{g} \vec{\nabla} T+\rho \sum_{k \in \Omega_{G}} h_{s, k} Y_{k} \vec{V}_{k}=-\lambda^{g} \vec{\nabla} T+\sum_{k \in \Omega_{G}} h_{s, k} \vec{J}_{k}
$$

With :

$$
J_{k, i}=\rho V_{k, i} Y_{k}=-\rho\left(D k \frac{W_{k}}{W} \frac{\partial X_{k}}{\partial x_{i}}-Y_{k} V_{i}^{\text {correc }}\right)
$$

Transport coefficients :

Temperature dependence is assumed for viscosity through a power law function. Non-binary Gas-phase species mass diffusion coefficients and thermal conductivity noted respectively $D_{k}$ and $\lambda^{g}$ are determined using constant Schmidt and Prandtl numbers which are considered to be constant in time and space as follows : 


$$
\begin{gathered}
D_{k}=\frac{\mu}{\rho S c_{k}} \\
\lambda^{g}=\frac{\mu C_{p}}{P_{r}}
\end{gathered}
$$

Species diffusion through temperature gradients known as Soret effect and the Dufour effect which is the heat flux induced by species mass fraction gradients are not accounted for in the present code [59, 60]. These assumptions that consist in neglecting the Soret and Dufour effects are verified thanks to a priori calculations using the EGlib library in Section 3.2.5.

Gas-phase kinetics :

The rate of production/consumption of the $k^{\text {th }}$ species noted $\dot{\omega}_{k}$ corresponds to the sum of rates $\dot{\omega}_{k j}$ produced by all gas-phase reactions :

$$
\dot{\omega}_{k}=\sum_{j \in \mathcal{M}} \dot{\omega}_{k j}=W_{k} \sum_{j \in \mathcal{M}}\left(v_{k j}^{\prime \prime}-v_{k j}^{\prime}\right) Q_{j}
$$

The progress rate $Q_{j}$ of reaction $j$ reads :

$$
Q_{j}=k_{f j} \prod_{k \in \Omega_{G}}\left(\rho Y_{k} / W_{k}\right)^{v_{k j}^{\prime}}-k_{r j} \prod_{k \in \Omega_{G}}\left(\rho Y_{k} / W_{k}\right)^{v_{k j}^{\prime \prime}}
$$

$k_{f j}$ and $k_{r j}$ are forward and reverse rate constants. The former is modeled using Arrhenius law whereas the latter is computed from the forward rates and the equilibrium constants.

The gas phase heat release rate reads :

$$
\dot{\omega}_{T}=-\sum_{k \in \Omega_{G}} \Delta h_{f, k}^{o} \dot{\omega}_{k}
$$

\section{Appendix B.}

In this appendix, the approach enabling to obtain mass and energy balance is detailed. The mass balance allows to obtain the expression of the Stefan velocity and the gas-phase species normal gradients accounting for surface reactions whereas the energy balance at the interface gives the normal heat flux at the reactive boundary. Flux balances at the reactive interface are obtained by integrating the transport equations over the control volume sketched in Fig. (B.20) :

\section{Control volume $\vartheta$}

with a surface
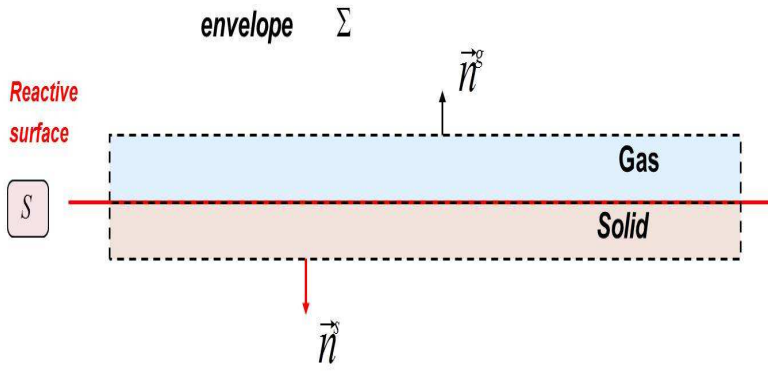

Figure B.20: sketch of the control volume at the reactive interface

$g$ is the index standing for gas-phase scalars whereas $s$ stands for solid-phase scalars. $w$ is the index standing for variables at the wall, $\overrightarrow{n^{g}}$ is the vector normal to the interface and directed toward the gas-phase, $\overrightarrow{n^{s}}$ is vector normal to the interface and directed toward the solid, $S$ is the elementary reactive surface which is also the fluid/structure interface, $\mathcal{V}$ refers to the control volume and $\Sigma$ stands for the surface envelope of the control volume. 


\section{Appendix B.1. Mass balance at a reactive interface}

The balance is obtained as follows : The species conservation Eq. (A.3) is integrated over the control volume $\mathcal{V}[26,61]$ and the Green-Ostrogradski theorem is applied :

$$
\begin{aligned}
\int_{\mathcal{V}}\left(\frac{\partial \rho Y_{k}}{\partial t}\right) d \mathcal{V}+\int_{\mathcal{V}}-\dot{\omega}_{k} d \mathcal{V} & =\int_{\Sigma}\left(-\left(\rho \overrightarrow{V_{k}} Y_{k}\right) \cdot \vec{n}\right) d \Sigma \\
& +\int_{\Sigma}-\left(\rho Y_{k} \vec{u} \cdot \vec{n}\right) d \Sigma
\end{aligned}
$$

Eq. (B.1) represents the integral form of the transport equation given in Eq. (A.3). If the volume tends to zero $(\mathcal{V} \rightarrow 0)$, the control volume surface $\Sigma$ tends to the reactive interface $S(\Sigma \rightarrow S)$ [61]. Since no mass accumulation occurs in a volume that tends to zero, the first term of the L.H.S of Eq. (B.1) becomes :

$$
\lim _{\mathcal{V} \rightarrow 0} \int_{\mathcal{V}}\left(\frac{\partial \rho Y_{k}}{\partial t}\right) d \mathcal{V}=0
$$

When surface chemistry occurs at the wall, one can decompose the gas-phase production rates into two terms, the first one would be non-zero in the gas-phase and null at the wall whereas the second one would equal zero in the gas-phase and non-zero at the solid/fluid interface as follows :

$$
\dot{\omega}_{k}=\dot{\omega}_{k, w}+\dot{s}_{k} \delta(y)
$$

where $\dot{\omega}_{k, w}$ and $\dot{s}_{k}$ represent respectively, for each $k^{t h}$ gas-pahse species, the gas-phase rate of production at the wall and the rate of production due to surface reactions per unit area. The dirac function integration gives :

$$
\int_{-\infty}^{+\infty} \delta(y) d y=1
$$

When the volume tends to zero, one can write :

$$
\lim _{\mathcal{V} \rightarrow 0} \int_{\mathcal{V}} \dot{\omega}_{k, w} d \mathcal{V}=0
$$

According to the Dirac function definition above :

$$
\lim _{\mathcal{V} \rightarrow 0} \int_{\mathcal{V}} \dot{\omega}_{k} d \mathcal{V}=\int_{S} \dot{s}_{k} d S
$$

Eventually, the volume integral of the L.H.S of Eq. (B.1) becomes :

$$
\lim _{\mathcal{V} \rightarrow 0}\left(\int_{\mathcal{V}}\left(\frac{\partial \rho Y_{k}}{\partial t}\right) d \mathcal{V}+\int_{\mathcal{V}}-\dot{\omega}_{k} d \mathcal{V}\right)=-\int_{S} \dot{s}_{k} d S
$$

Since there are no convection and diffusion in the solid and the boundary of the control volume is static $\left(\vec{J}_{k}^{s}=\vec{u}^{s}=\overrightarrow{0}\right)$, one obtains for the R.H.S of Eq. (B.1) :

$$
\begin{aligned}
& \lim _{\Sigma \rightarrow S}\left(\int_{\Sigma}\left(-\left(\rho \vec{V}_{k} Y_{k}\right) \cdot \vec{n}\right) d \Sigma+\int_{\Sigma}\left(\rho Y_{k}(-\vec{u}) \cdot \vec{n}\right) d \Sigma\right)= \\
& \int_{S}\left(-\left(\rho_{w}^{g} \vec{V}_{k, w}^{g} Y_{k, w}\right) \cdot \vec{n}^{g}\right) d S+\int_{S}\left(\rho_{w}^{g} Y_{k, w}\left(-\vec{u}^{g}\right) \cdot \vec{n}^{g}\right) d S
\end{aligned}
$$

As a result, Eqs.(B.7, B.8) give : 


$$
\begin{aligned}
\underbrace{-\int_{S} \dot{s}_{k} d S}_{\text {oduction by surface reaction }}= & -\underbrace{\int_{S}\left(\rho_{w}^{g} \vec{V}_{k, w}^{g} Y_{k, w}\right) \cdot \vec{n}^{g} d S}_{\text {Mass flux diffusion }} \\
& +\underbrace{\int_{S} \rho_{w}^{g} Y_{k, w}\left(-\vec{u}^{g} \cdot \vec{n}^{g}\right) d S}_{\text {Mass flux convection }}
\end{aligned}
$$

Where :

$$
\left\{\begin{aligned}
\vec{V}_{k, w}^{g} \cdot \vec{n}^{g} & =V_{k, n, w} \\
\vec{u} \cdot \vec{n}^{g} & =U_{\text {Stefan }}
\end{aligned}\right.
$$

The mass balance at the reactive interface (Eq. (B.9)) reads then :

$$
\left(\rho_{w} V_{k, n, w} Y_{k, w}\right)+\left(\rho_{w} U_{\text {Stefan }} Y_{k, w}\right)=\dot{s}_{k}
$$

Eq. (B.10) is consistent with the mass balance near a reactive wall in $[20,26,37,62]$. Summing Eq. (B.10) over all the gas-phase species and considering the mass conservation, an expression for the wall-normal Stefan velocity is obtained:

$$
U_{\text {Stefan }}=\frac{1}{\rho_{w}}\left(\sum_{k \in \Omega_{G}} \dot{s}_{k}\right)
$$

Appendix B.1.1. Gas-phase species mass fraction

$$
\text { gradient correction }
$$

This section shows how the prescribed gradient is written. First, let us consider the mass balance at the interface given by Eq. (B.10) demonstrated in the previous Section Appendix B.1:

$$
\left(\rho_{w} V_{k, n, w} Y_{k, w}\right)+\left(\rho_{w} U_{\text {Stefan }} Y_{k, w}\right)=\dot{s}_{k}
$$

By isolating the species diffusion flux, one obtains:

$$
\left(\rho_{w} V_{k, n, w} Y_{k, w}\right)=\dot{s}_{k}-\left(\rho_{w} U_{\text {Stefan }} Y_{k, w}\right)
$$

The species mass flux is written as in Eq. (A.7) according to the Hirschfelder \& Curtiss approximation:

$$
J_{k, i}=\rho V_{k, i} Y_{k}=-\rho\left(D k \frac{W_{k}}{W} \frac{\partial X_{k}}{\partial x_{i}}-Y_{k} V_{i}^{\text {correc }}\right)
$$

By multiplying Eq. (B.14) by the interface normal $\vec{n}^{g}$, one obtains :

$$
\begin{aligned}
\vec{J}_{k, w} \cdot \vec{n}^{g} & =\rho_{w} \vec{V}_{k, w} \cdot \vec{n}^{g} Y_{k, w}= \\
& -\rho_{w}\left(D k \frac{W_{k}}{W_{w}} \vec{\nabla} X_{k, w} \cdot \vec{n}^{g}-Y_{k, w} \vec{V}_{w}^{\text {correc }} \cdot \vec{n}^{g}\right)
\end{aligned}
$$

with $\vec{V}_{w}^{\text {correc }} \cdot \vec{n}^{g}$ and $\vec{\nabla} X_{k, w} \cdot \vec{n}^{g}$ corresponding respectively to the wall-orthogonal correction velocity and species molar fractions:

$$
\vec{V}_{w}^{\text {correc }} \cdot \vec{n}^{g}=\sum_{k \in \Omega_{G}} D_{k} \frac{W_{k}}{W_{w}} \vec{\nabla} X_{k, w} \cdot \vec{n}^{g}
$$

$$
\vec{\nabla} X_{k, w} \cdot \vec{n}^{g}=\frac{W_{w}}{W_{k}} \vec{\nabla} Y_{k, w} \cdot \vec{n}^{g}-\frac{W_{w}^{2}}{W_{k}} Y_{k, w} \sum_{l \in \Omega_{G}} \frac{1}{W_{l}} \vec{\nabla} Y_{l, w} \cdot \vec{n}^{g}
$$

Inserting Eqs. (B.11, B.16, B.15) into the mass balance at the interface given by Eq. (B.13), it becomes possible to relate the species mass fraction at the wall $Y_{k, w}$ to its 
normal gradient at the boundary surface by isolating the latter

$$
\begin{aligned}
\vec{\nabla} Y_{k, w}^{B C} \cdot \vec{n}^{g} & =\frac{Y_{k, w}}{D_{k} \rho_{w}} \sum_{l \in \Omega_{G}} \dot{s}_{l}+\frac{Y_{k, w}}{D_{k}} \vec{V}_{w}^{\text {correc }} \cdot \vec{n}^{g} \\
& +Y_{k, w} W_{w}\left(\sum_{l \in \Omega_{g a s}} \frac{1}{W_{l}} \vec{\nabla} Y_{k, w} \cdot \vec{n}^{g}\right)-\frac{\dot{s}_{k}}{\rho_{w} D_{k}}
\end{aligned}
$$

Appendix B.2. Energy balance at the reactive interface and heat flux correction

An energy balance at the reactive interface has been developed in order to prescribe the proper temperature gradient at the wall. The same approach used for the mass balance is applied to the total non-chemical energy given in Eq. (A.2). By integrating over the control volume, applying the Green-Ostogradski theorem to Eq. (A.2) and tending the volume to zero, one obtains:

$$
\begin{aligned}
& \lim _{\mathcal{V} \rightarrow 0}\left(\int_{\mathcal{V}} \frac{\partial \rho E}{\partial t} d \mathcal{V}+\int_{\mathcal{V}}-\dot{\omega}_{T} d \mathcal{V}\right)= \\
& \lim _{\Sigma \rightarrow S}\left(\int_{\Sigma}((\overline{\bar{\sigma}} \vec{u}-\vec{q}) \cdot \vec{n}) d \Sigma+\int_{\Sigma}(-\rho E \vec{u} \cdot \vec{n}) d \Sigma\right)
\end{aligned}
$$

As shwon in Eqs. (B.2-B.5) the fisrt term of the L.H.S equals zero and the second term corresponds to the heat-release due surface reactions. Regarding the R.H.S, the same approach as in Eq. (B.8) is used thus giving the heat balance at the reactive interface :

$$
-\dot{S}_{T}=\left(\overline{\bar{\sigma}}^{g} \cdot \vec{u}^{g}\right)_{n, w}-q_{n, w}^{g}-\rho_{w}^{g} E_{w} U_{S t e f a n}+q_{n, w}^{s}
$$

With the following notations :

$$
\begin{aligned}
\int_{S} \dot{S}_{T} d S & =\lim _{\mathcal{V} \rightarrow 0}\left(\int_{\mathcal{V}} \dot{\omega}_{T} d \mathcal{V}\right) \\
\left(\overline{\bar{\sigma}}^{g} \cdot \vec{u}^{g}\right)_{n, w}-q_{n, w}^{g} & =\left(\overline{\bar{\sigma}}_{w}^{g} \vec{u}_{w}^{g}-\vec{q}_{w}^{g}\right) \cdot \vec{n}^{g} \\
U_{\text {Stefan }} & =\vec{u}_{w} \cdot \vec{n}^{g} \\
q_{n, w}^{s} & =\left(-\vec{q}_{w}^{s}\right) \cdot \vec{n}^{g}
\end{aligned}
$$

If one considers that there is no species diffusion in the solid phase so that only solid thermal diffusion remains and that the surface heat release is due to both solid-phase and gas-phase species :

$$
\left\{\begin{aligned}
q_{n, w}^{s} & =-\lambda^{s} \vec{\nabla} T_{w}^{s} \cdot \vec{n}^{g} \\
\dot{S}_{T} & =-\left(\sum_{k \in \Omega_{S}} h_{k} \dot{s}_{k}+\sum_{k \in \Omega_{G}} \Delta h_{f, k}^{o} \dot{s}_{k}\right)
\end{aligned}\right.
$$

By introducing the terms above into Eq. (B.20), and replacing $E$ by its expression one obtains :

$$
\begin{aligned}
& \left(\lambda^{g} \vec{\nabla} T_{w}^{g}-\lambda^{s} \vec{\nabla} T_{w}^{s}\right) \cdot \vec{n}^{g}= \\
& +\sum_{k \in \Omega_{G}}\left(\rho_{w}\left(h_{s, k, w} V_{k, n, w} Y_{k, w}\right)+\rho_{w} U_{\text {Stefan }}\left(h_{s, k, w} Y_{k, w}\right)\right) \\
& +\left(\sum_{k \in \Omega_{S}} h_{k, w} \dot{s}_{k}+\sum_{k \in \Omega_{G}} \Delta h_{f}^{o} \dot{s}_{k}\right) \\
& -\left(\left(\overline{\bar{\sigma}}^{g}{\overrightarrow{u^{g}}}_{n, w}+p U_{\text {Stefan }}\right)\right. \\
& +\frac{\dot{m}}{2} \sum_{j \in \mathcal{D}} u_{j, w}^{2}
\end{aligned}
$$

The first term in the R.H.S of Equation Eq. (B.21) can be simplified by considering the mass balance at the 
interface given in Eq. (B.10). Indeed, if one multiplies Eq. (B.10) by the species sensible enthalpy $h_{s, k}$ and sums all over the gas-phase species, the resulting balance becomes :

$$
\begin{aligned}
& \sum_{k \in \Omega_{G}}\left(\rho_{w}\left(h_{s, k, w} V_{k, n, w} Y_{k, w}\right)+\rho_{w} U_{\text {Stefan }}\left(h_{s, k, w} Y_{k, w}\right)\right)= \\
& \sum_{k \in \Omega_{G}} h_{s, k, w} \dot{s}_{k}
\end{aligned}
$$

As a result, the first and second terms in the R.H.S of equation Eq. (B.21) are simplified as follows :

$$
\begin{aligned}
& \sum_{k \in \Omega_{G}}\left(\rho_{w}\left(h_{s, k, w} V_{k, n, w} Y_{k, w}\right)+\rho_{w} U_{\text {Stefan }}\left(h_{s, k, w} Y_{k, w}\right)\right) \\
& +\left(\sum_{k \in \Omega_{S}} h_{k, w} \dot{s}_{k}+\sum_{k \in \Omega_{G}} \Delta h_{f}^{o} \dot{s}_{k}\right)=\sum_{k \in\left\{\Omega_{G} \cup \Omega_{S}\right\}} h_{k, w} \dot{s}_{k}
\end{aligned}
$$

and the third term becomes:

$$
\begin{aligned}
\left(\left(\overline{\bar{\sigma}}^{g} \vec{u}^{g}\right)_{n, w}+p U_{\text {Stefan }}\right) & =\left(\left(\overline{\bar{\sigma}}^{g} \vec{u}^{g}\right)_{n, w}+p \vec{u}^{g} \cdot \vec{n}^{g}\right) \\
& =\left(\overline{\bar{\tau}}^{g} \vec{u}^{g}\right)_{n, w}
\end{aligned}
$$

Eventually, injecting Eqs. (B.23, B.24) into Eq. (B.21), and isolating the gas-phase wall-normal thermal diffusion flux, yields :

$$
\begin{aligned}
\lambda^{g} \vec{\nabla} T_{w}^{g} \cdot \vec{n}^{g} & =\sum_{k \in\left\{\Omega_{G} \cup \Omega_{S}\right\}} h_{k, w} \dot{s}_{k}-\left(\overline{\bar{\tau}}^{g} \vec{u}^{g}\right)_{n, w} \\
& +\frac{\dot{m}}{2} \sum_{j \in \mathcal{D}} u_{j, w}^{2}+\lambda^{s} \vec{\nabla} T_{w}^{s} \cdot \vec{n}^{g}
\end{aligned}
$$

Eq. (B.25) constitutes the heat flux balance that accounts for the effect of surface chemistry.

\section{Appendix B.2.1. Heat flux correction}

The heat flux balance of Eq. (B.25) balance enables to write the heat flux to be prescribed at the boundary condition $\overrightarrow{q_{w}}{ }^{B C} \cdot \vec{n}^{g}$ by isolating in Eq. (B.25) the heat flux $q_{j}$ given in Eq. (A.6). Under the Hirschfelder \& Curtiss approximation ${\overrightarrow{q_{w}}}^{B C} \cdot \vec{n}^{g}$ reads :

$$
\begin{aligned}
& \vec{q}_{w}^{B C} \cdot \vec{n}^{g}=\quad-\underbrace{\sum_{k \in \Omega_{S}} h_{k} \dot{s}_{k}} \\
& \text { Surface heat release due to surface species } \\
& -\underbrace{\sum_{k \in \Omega_{G}} \Delta h_{f}^{o} \dot{s}_{k}}_{\text {Surface heat release due to gas-phase species }} \\
& -\underbrace{\rho_{w} \sum_{k \in \Omega_{G}} U_{S t e f a n} h_{s, k, w} Y_{k, w}}_{\text {Sensible enthalpy transport by Stefan flux }} \\
& -\underbrace{\frac{\dot{m}}{2} \sum_{j \in \mathcal{D}} u_{j, w}^{2}}_{\text {Kinetic energy }}+\underbrace{\left(\overline{\bar{\tau}}^{g} \overrightarrow{u^{g}}\right)_{n, w}}_{\text {Viscous heat }}-\underbrace{\lambda^{s} \vec{\nabla} T_{w}^{s} \cdot \vec{n}^{g}}_{\text {Solid heat diffusion }}
\end{aligned}
$$

\section{Appendix C.}

Making use of the assumption of steady developed non-reacting flow, one can write mass/species/momentum conservation equations as:

$$
\begin{gathered}
\frac{\partial(\rho v)}{\partial y}=0 \\
\frac{\partial}{\partial y}\left(\rho\left(v+V_{k, y}\right) Y_{k}\right)=0, \quad k \in\{1,2\} \\
\frac{\partial(\rho u v)}{\partial y}=\frac{\partial \tau_{x y}}{\partial y}+\mathcal{S}
\end{gathered}
$$

$$
\frac{p}{\rho}=\frac{R}{W} T
$$


The momentum source term $S$ is used for the following numerical and paractical reasons:

- Standard Inlet/Outlet boundary conditions imply the estimation of a pressure loss in the streamwise direction to include in the momentum conservation equation which is not an accurate approach for analytical solution derivation and not strictly conservative.

- Periodicity enables momentum conservation by imposing a momentum source thus being strictly conservative.

In the following, the letter $C, O$ and $P$ denote the solid wall species (which could be for instance solid carbon), the gaseous oxidizing species and the gaseous species produced by gasification, respectively. The associated stoichiometric coefficients are noted $v_{C}, v_{O}$ and $v_{P}$. According to these notations, the following chemical schemes are applied to the surface 1 (gasification):

$$
v_{C} C_{(s)}+v_{O} O \rightarrow v_{P} P
$$

and to the surface 2 (lieu of deposition):

$$
v_{P} P \rightarrow v_{C} C_{(s)}+v_{O} O
$$

In what follows, variables subscripted by letters $C$, $O$ and $P$ refer to the associated species and subscript numbers 1 and 2 refer to space location, $y=0$ and $y=h$, respectively. The molar progress rate of reaction $1, Q_{1}$, can then be modeled as follows:

$$
Q_{1}=\left(\frac{Y_{O, 1}}{W_{O}} \rho_{1}\right)^{v_{O}} K_{1}=\left(X_{O, 1} \frac{p}{R T}\right)^{v_{O}} K_{1}
$$

where $K_{1}$ represents the rate of reaction 1 which can be modeled by an Arrhenius law for instance. However, in the present framework it is not necessary to go deeper in the modeling of this coefficient because one assumes a constant temperature implying that $K_{1}$ can be merely seen as a parameter of the problem. Similarly, one can write the molar progress rate of reaction 2 as:

$$
Q_{2}=\left(\frac{Y_{P, 2}}{W_{P}} \rho_{2}\right)^{v_{P}} K_{2}=\left(X_{P, 2} \frac{p}{R T}\right)^{v_{P}} K_{2}
$$

Moreover, the general formulation of species conservation balance at the reacting surfaces reads:

$$
\left(\rho_{j} \vec{u} Y_{k}+\rho \vec{V}_{k} Y_{k}\right) \cdot \vec{n}=\dot{s}_{k}
$$

The species conservation balance at surface 1 reads:

$$
\rho_{1} v_{1} Y_{k, 1}+\rho_{1} V_{k, 1} Y_{k, 1}=\dot{s}_{k, 1}
$$

and at surface 2 :

$$
\rho_{2} v_{2} Y_{k, 2}+\rho_{2} V_{k, 2} Y_{k, 2}=-\dot{s}_{k, 2}
$$

where $\vec{n}$ is the vector normal to the wall and pointing toward the gas-phase. Summing over all the species and making use of the mass conservation constraints $\sum_{k} Y_{k}=1$ and $\sum_{k} Y_{k} V_{k}=0$, one obtains the two relations $\rho_{1} v_{1}=\sum_{k} \dot{s}_{k, 1}$ and $\rho_{2} v_{2}=-\sum_{k} \dot{s}_{k, 2}$. Furthermore, the continuity equation Eq. (C.1) imposes that $\rho(y) v(y)$ is constant (in the forthcoming the notation $\dot{m}=\rho(y) v(y)$ will be used). As a consequence, one finds that $\dot{m}=\sum_{k} \dot{s}_{k, 1}=-\sum_{k} \dot{s}_{k, 2}$ which leads to the relation $Q_{1}=Q_{2}$ (the notation $Q=Q_{j}$ is thus retained 
for simplification). The two progress rates of the surface reactions are thus identical. The relation between the wall normal mass flow rate and the progress rate of reaction is thus expressed as:

$$
\dot{m}=Q\left(v_{P} W_{P}-v_{O} W_{O}\right)
$$

When the species diffusion due to pressure gradients, temperature gradients (Soret effect) and volume forces are neglected, the binary diffusion between the two species $O$ and $P$ can be modeled by the classical relation [35]

$V_{k} Y_{k}=-\mathcal{D}_{O P}^{\text {bin }} \partial Y_{k} / \partial y$, where $\mathcal{D}_{O P}^{\text {bin }}=\mathcal{D}_{P O}^{\text {bin }}$ is the binary diffusion coefficient. Another formulation is obtained by considering equivalent diffusion coefficients, $D_{k}$, that represents the diffusion of species $k$ into the mixture and expressed as $D_{k}=\left(1-Y_{k}\right) /\left(\sum_{l \neq k} X_{l} / \mathcal{D}_{k l}^{\text {bin }}\right)$. This leads to an equivalent diffusion model which now involves the species molar fractions instead of their mass fractions, $V_{k} X_{k}=-D_{k} d X_{k} / d y$. This presents a strong advantage for solving the system of equations. Note also that contrary to the binary coefficient, $D_{k}$ is depending on the local concentration of each species which means that $D_{k}=D_{k}(y)$. According to the previous definitions, the following relation stands for the present mixture:

$$
D_{O} W_{O}=D_{P} W_{P}
$$

Moreover, the dynamic viscosity for gases is usually assumed to be solely temperature dependent which means that in the present isothermal framework $\mu$ is a constant. Since the $S c h m i d t$ numbers, $S c_{k}=$ $\mu /\left(\rho D_{k}\right)$, are assumed to be constant, we find that $\rho(y) D_{k}(y)$ is also constant. This statement allows to simplify the integration of the forthcoming partial differential equations. In order to determine the species concentration profiles, one may concentrate on the variable $X_{O}$ and then deduce the concentration of species $P$ by the relation $X_{P}=1-X_{O}$. At this step, it is important to consider molar fractions instead of mass fractions because it leads to a first order partial differential equation with constant coefficients. Indeed, making use of Eq. (C.2), integrating once and identifying with Eq. (C.10), rearranging in terms of molar fractions, and making use of $\rho(y) D_{O}(y)=\mu / S c_{O}$ yields:

$$
\eta X_{O}-\frac{\mu}{S c_{O} \dot{m}} \frac{d X_{O}}{d y}=\frac{v_{O} W_{P}}{v_{O} W_{O}-v_{P} W_{P}}
$$

with

$$
\eta=\frac{W_{P}\left(v_{O}-v_{P}\right)}{v_{O} W_{O}-v_{P} W_{P}}
$$

Since $\eta=0$ for $v_{O}=v_{P}$, the integration of this differential equation leads to two solutions depending on the values taken by $v_{O}$ and $v_{P}$ :

$$
X_{O}(y)=\left(X_{O, 1}-\frac{v_{O}}{v_{O}-v_{P}}\right) \exp \left[\eta \frac{S c_{O} \dot{m}}{\mu} y\right]+\frac{v_{O}}{v_{O}-v_{P}}
$$

for $v_{O} \neq v_{P}$

$X_{O}(y)=-\frac{S c_{O} \dot{m}}{\mu} \frac{W_{P}}{W_{O}-W_{P}} y+X_{O, 1}$

for $v_{O}=v_{P}$ 
where $X_{O, 1}$ is the molar fraction of species $O$ at surface 1. The value of this constant is determined thanks to the relation between $X_{O, 1}$ and $X_{O, 2}$ that comes from the identity $Q_{1}=Q_{2}$ which according to Eqs. (C.7, C.8) gives:

$$
X_{O, 1}^{v_{O}}\left(\frac{p}{R T}\right)^{v_{O}-v_{P}} \frac{K_{1}}{K_{2}}=\left(1-X_{O, 2}\right)^{v_{P}}
$$

Furthermore, injecting Eq. (C.7) into the wall normal mass flow rate Eq. (C.12) yields the dependency relation between $\dot{m}$ and $X_{O, 1}$

$$
\dot{m}=X_{O, 1}^{v_{O}}\left(\frac{p}{R T}\right)^{v_{O}} K_{1}\left(v_{P} W_{P}-v_{O} W_{O}\right)
$$

Hence, Eq. (C.16) expressed for $y=h$ can be injected into Eq. (C.17), and with the use of Eq. (C.18) one obtains an equation in which $X_{O, 1}$ is the only unknown. This manipulation allows determining the $X_{O, 1}$ constant and Eqs. (C.15-C.18) form the analytical solution for the molar fraction of species $O$. The molar fraction of $P$ is then given by the relation $X_{P}(y)=1-$ $X_{O}(y)$. Concerning the determination of the streamwise velocity profile, one introduces the newtonian fluid relation $\tau_{x y}=\mu d u / d y$ into Eq. (C.3) which gives $\rho v d u / d y-\mu d^{2} u / d y^{2}=\mathcal{S}$. Since $\mu$ is a constant under the approximation of constant temperature, and because $\rho(y) v(y)=\dot{m}$ is a constant according to the continuity Eq. (C.1), the latter differential equation is merely of second order with constant coefficients. Its integration with the boundary conditions $u(0)=u(h)=0$ leads to the following solution:

$$
u(y)=\frac{\mathcal{S}}{\dot{m}}\left[\frac{h}{\mathrm{e}^{\frac{\dot{m}}{\mu} h}-1}\left(1-\mathrm{e}^{\frac{\dot{m}}{\mu} y}\right)+y\right]
$$

where $\dot{m}$ is determined from Eq. (C.18). The maximum streamwise velocity is expected to be observed at the wall normal distance:

$$
\left.y\right|_{u_{\max }}=\frac{\mu}{\dot{m}} \ln \left[\frac{\mu}{h \dot{m}}\left(\mathrm{e}^{\frac{\dot{m}}{\mu} h}-1\right)\right]
$$

